Rev. MAt. Iberoamericana 21 (2005), no. 3, 771-818

\title{
Potential Theory for Schrödinger operators on finite networks
}

Enrique Bendito, Ángeles Carmona and Andrés M. Encinas

\begin{abstract}
We aim here at analyzing the fundamental properties of positive semidefinite Schrödinger operators on networks. We show that such operators correspond to perturbations of the combinatorial Laplacian through 0-order terms that can be totally negative on a proper subset of the network. In addition, we prove that these discrete operators have analogous properties to the ones of elliptic second order operators on Riemannian manifolds, namely the monotonicity, the minimum principle, the variational treatment of Dirichlet problems and the condenser principle. Unlike the continuous case, a discrete Schrödinger operator can be interpreted as an integral operator and therefore a discrete Potential Theory with respect to its associated kernel can be built. We prove that the Schrödinger kernel satisfies enough principles to assure the existence of equilibrium measures for any proper subset. These measures are used to obtain systematic expressions of the Green and Poisson kernels associated with Dirichlet problems.
\end{abstract}

\section{Introduction}

In the last years, a considerable amount of works that extend properties and results in elliptic boundary value problems on Riemannian manifolds to the graph framework have been published. Frequently, the discrete structure allows to obtain the fundamental features without the technicalities that darken the results. Moreover, the simplicity of the discrete setting enables to use successfully tools that do not seem to have a continuous counterpart.

2000 Mathematics Subject Classification: 31C20, 34B45.

Keywords: Combinatorial Laplacian, Schrödinger operators, Dirichlet forms, Green kernel, Poisson kernel, Discrete Potential Theory, equilibrium measures, effective resistance. 
In this paper we analyze the fundamental properties of a general class of Schrödinger operators on networks that includes those whose associated quadratic form is positive semidefinite but it is not a Dirichlet form. Specifically, we study the monotonicity properties of this kind of discrete operators, the variational treatment of Dirichlet problems and the properties of their resolvent kernels. Besides, we take advantage of considering the Schrödinger operator as an integral operator and then we build a Discrete Potential Theory for the (signed) kernel of such integral operator.

A Schrödinger operator on a finite network is an operator of the form $\mathcal{L}_{q}=\mathcal{L}+q$, where $\mathcal{L}$ is the combinatorial Laplacian of the network and $q$ is a function on the vertex set. So, a Schrödinger operator can be seen as a perturbation of the combinatorial Laplacian. It is well-known that the quadratic form associated with this operator is a Dirichlet form if and only if $q$ is non-negative, [8]. Conversely, any Dirichlet form over a finite space, $V$, can be seen as the Dirichlet form associated with a Schrödinger operator with non negative 0 -order term on a network that has $V$ as vertex set. Here, we study under which condition the quadratic form associated with a Schrödinger operator is positive semidefinite and we show that this condition guarantees that an important family of contractions operates with respect to it.

The monotonicity properties of Schrödinger operators are well-known in the case $q \geq 0$, see for instance [14, 20]. In this paper we extend the above results to the case when $\mathcal{L}_{q}$ is only positive semidefinite. We also prove a version of a discrete Hopf's minimum principle, that has not a continuous counterpart when $q$ is non positive.

The Potential Theory associated with Dirichlet Forms has been extensively treated in the literature, see for instance $[2,14,20]$. Alternatively, for the standard case in which $q \geq 0$, the authors developed a Potential Theory with respect to the kernel associated with the operator $\mathcal{L}_{q}$ when it is seen as an integral operator, see [4]. Here we extend these techniques when $\mathcal{L}_{q}$ is positive semidefinite. This tool, that has not a continuous equivalent, allows to obtain systematically explicit expressions of the resolvent kernels.

The paper is organized as follows. In Section 2 we give the basic concepts and notations on networks and we analyze the functions that can be used to perturb the combinatorial Laplacian in such a way that the corresponding Schrödinger operator be positive semidefinite. In particular, we show that for any proper subset we can consider functions of this type that are totally negative on it. Moreover, we prove that the lower bound of such functions depends only on the network geometry.

The properties of the quadratic form associated with positive semidefinite Schrödinger operators are studied in Section 3. We show that they 
are characterized for being non increasing with respect to a transformation determined by the ground state, that is, the positive eigenfunction associated to the minimum eigenvalue of the Schrödinger operator. Moreover, we obtain that each quadratic form of this type takes the same values as the Dirichlet form associated with a Schrödinger operator on a new network whose conductances are given by the conductances of the original network and by the value of the ground state.

In Section 4 we show that the monotonicity property is equivalent to a version of a general minimum principle under the hypothesis of positive semidefiniteness of the Schrödinger operators. As a consequence, we establish a generalization of the well-known Condenser principle where the prescribed value at the positive plate is given by the ground state.

In Section 5 we study the Green and Poisson kernels associated to Dirichlet problems. We determine the relation between them and we show that their properties are similar to the ones verified by the analogous kernels in the continuous case. We also build the generalized Green kernels associated with a singular Schrödinger operator. In addition we show that, in this framework, an elementary discrete version of the Schwartz's Kernel Theorem makes sense, which allows us to consider the kernel associated with a positive semidefinite Schrödinger operator. In the last section, we investigate the properties of these kernels in the context of the Discrete Potential Theory. We prove that the Schrödinger kernel satisfies the equilibrium principle which, in particular, enables us to obtain explicit expressions of the Green and Poisson kernel in terms of equilibrium measures.

\section{Preliminaries}

Let $\Gamma=(V, E, c)$ be a finite network, that is, a finite connected graph without loops nor multiple edges, with vertex set $V$ and edge set $E$, in which each edge $(x, y)$ has been assigned a conductance $c(x, y)>0$. Moreover, $c(x, y)=c(y, x)$ and $c(x, y)=0$ if $(x, y) \notin E$. We say that $x$ is adjacent to $y, x \sim y$, if $(x, y) \in E$ and for all $x \in V$, the value

$$
k(x)=\sum_{y \in V} c(x, y)
$$

is called total conductance at $x$ or degree of $x$. A path of length $m \geq 1$ is a sequence $\left\{x_{1}, \ldots, x_{m+1}\right\}$ of vertices such that $c\left(x_{i}, x_{i+1}\right)>0$, or equivalently $x_{i} \sim x_{i+1}, i=1, \ldots, m$. That $\Gamma$ is connected means that any two vertices of $V$ can be joined by a path. More generally, a subset $F$ of $V$ is said to be connected if each pair of vertices of $F$ is joined by a path entirely contained in $F$. If $x \neq y$, we denote by $d(x, y)$ the minimum length between the paths 
joining $x$ and $y$. Defining $d(x, x)=0$, the application $d$ determines a metric on $V$ whose associated topology is the discrete one. For this reason we will prefer to use geometric concepts instead of topological ones. So, given $F \subset V$, we denote by $F^{c}$ its complementary in $V$ and we call interior, vertex boundary, closure and exterior of $F$ the subsets

$$
\begin{aligned}
\stackrel{\circ}{F} & =\{x \in F: y \in F \text { for all } y \sim x\}, \\
\delta(F) & =\{x \in V: d(x, F)=1\}, \\
\bar{F} & =\{x \in V: d(x, F) \leq 1\} \quad \text { and } \\
\operatorname{Ext}(F) & =\{x \in V: d(x, F) \geq 2\},
\end{aligned}
$$

respectively. Observe that when $F \neq \emptyset$ unlike the topological case,

$$
\delta(F) \cap \delta\left(F^{c}\right)=\emptyset \quad \text { and } \quad \bar{F}=F \text { or } \stackrel{\circ}{F}=F \text { iff } F=V .
$$

However, the following relations, which are similar to the topological ones, are satisfied:

$$
\begin{aligned}
& \bar{F}=F \cup \delta(F), \quad F=\delta\left(F^{c}\right) \cup \stackrel{\circ}{F}, \\
& \delta\left(F^{c}\right) \cap \stackrel{\circ}{F}=\emptyset, \quad \delta(\stackrel{\circ}{F}) \subset \delta\left(F^{c}\right) \quad \text { and } \quad \operatorname{Ext}(F)=V \backslash \bar{F} .
\end{aligned}
$$

Moreover, when $F$ is connected then $\bar{F}$ is also connected, but $\stackrel{\circ}{F}$ is not connected, in general.

The set of functions on $V$, denoted by $\mathcal{C}(V)$, and the set of non-negative functions on $V, \mathcal{C}^{+}(V)$, are naturally identified with $\mathbb{R}^{n}$ and the positive cone of $\mathbb{R}^{n}$, respectively, where $n=|V|$. If $u \in \mathcal{C}(V)$, its support is given by $\operatorname{supp}(u)=\{x \in V: u(x) \neq 0\}$. Moreover, if $F$ is a non empty subset of $V$, we consider the sets

$$
\begin{aligned}
\mathcal{C}(F) & =\{u \in \mathcal{C}(V): \operatorname{supp}(u) \subset F\} \\
\mathcal{C}^{+}(F) & =\mathcal{C}(F) \cap \mathcal{C}^{+}(V) \quad \text { and } \\
\mathcal{C}^{*}(F) & =\left\{u \in \mathcal{C}^{+}(F): \operatorname{supp}(u)=F\right\} .
\end{aligned}
$$

For each $F \subset V$, the characteristic function of $F$ will be denoted by $1_{F}$. When $F=V$ we will omit the subscript, whereas when $F=\{x\}$, its characteristic function will be denoted by $\varepsilon_{x}$.

Throughout the paper $d x$ will denote the counting measure on $V$ and hence for all $u \in \mathcal{C}(V)$,

$$
\int_{V} u d x=\sum_{x \in V} u(x) .
$$

In the sequel, we suppose that $\mathcal{C}(V)$ is endowed with the Hilbert space structure induced by $d x$ and for each $u \in \mathcal{C}(V)$ we will denote by $\|u\|_{2}$ the associated norm. 
The combinatorial Laplacian of $\Gamma$ is the linear operator $\mathcal{L}: \mathcal{C}(V) \longrightarrow \mathcal{C}(V)$ that assigns to each $u \in \mathcal{C}(V)$ the function

$$
\mathcal{L}(u)(x)=\int_{V} c(x, y)(u(x)-u(y)) d y .
$$

If $F$ is a proper subset of $V$, for each $u \in \mathcal{C}(\bar{V})$ we define the normal derivative of $u$ as the function in $\mathcal{C}(\delta(F))$ given by

$$
\left(\frac{\partial u}{\partial \mathrm{n}}\right)(x)=\int_{F} c(x, y)(u(x)-u(y)) d y,
$$

for all $x \in \delta(F)$, see for instance $[4,9]$. The relation between the values of the combinatorial Laplacian on $F$ and the values of the normal derivative at $\delta(F)$ is given by the Second Green Identity, proved in [4]

$$
\int_{F}(v \mathcal{L}(u)-u \mathcal{L}(v)) d x=\int_{\delta(F)}\left(u \frac{\partial v}{\partial \mathrm{n}}-v \frac{\partial u}{\partial \mathrm{n}}\right) d x, \text { for all } u, v \in \mathcal{C}(\bar{F}) .
$$

When $F=V$ the above identity tell us that the combinatorial Laplacian is a self-adjoint operator and that $\int_{V} \mathcal{L}(u) d x=0$ for any $u \in \mathcal{C}(V)$. Moreover, since $\Gamma$ is connected $\mathcal{L}(u)=0$ iff $u$ is a constant function.

A Schrödinger operator on $\Gamma$ is a linear operator $\mathcal{L}_{q}: \mathcal{C}(V) \longrightarrow \mathcal{C}(V)$ that assigns to each $u \in \mathcal{C}(V)$ the function

$$
\mathcal{L}_{q}(u)(x)=\mathcal{L}(u)(x)+q(x) u(x),
$$

where $q$ is an arbitrary function in $\mathcal{C}(V)$, see [11]. In an equivalent manner, a Schrödinger operator on $\Gamma$ is nothing but a 0 -order perturbation of the combinatorial Laplacian.

Observe that if $u \in \mathcal{C}(V)$ and $F=\operatorname{supp}(u)$, then $\mathcal{L}_{q}(u)=\mathcal{L}(u)$ on $F^{c}$. Moreover, if $u \in \mathcal{C}^{+}(V)$, then $\mathcal{L}_{q}(u)<0$ on $\delta(F)$ and $\mathcal{L}_{q}(u)=0$ on $\operatorname{Ext}(F)$. On the other hand, a Schrödinger operator is self-adjoint in the sense that

$$
\int_{V} v \mathcal{L}_{q}(u) d x=\int_{V} u \mathcal{L}_{q}(v) d x \quad \text { for all } u, v \in \mathcal{C}(V) .
$$

For fixed $q \in \mathcal{C}(V)$ and for each non empty subset $F$ of $V$, we consider the following boundary value problem: given $f \in \mathcal{C}(F)$ and $g \in \mathcal{C}(\delta(F))$ find $u \in \mathcal{C}(\bar{F})$ such that

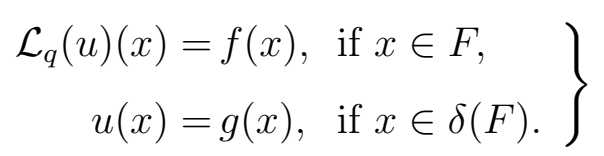

When $F \neq V$ this problem is known as Dirichlet problem on $F$, whereas if $F=V$ it is called Poisson equation on $V$. 
It is clear that when $F \neq V$, the Dirichlet problem on $F$ is equivalent to the following semi-homogeneous Dirichlet problem

$$
\left.\begin{array}{clrl}
\mathcal{L}_{q}(u)(x) & =f(x)-\mathcal{L}(g)(x), & & \text { if } x \in F, \\
u(x) & =0, & & \text { if } x \in \delta(F) .
\end{array}\right\} \quad[\mathrm{BVP}]_{0}
$$

So, for each non empty subset $F \subset V$, the study of existence and uniqueness of solution for [BVP] is reduced to the study of existence and uniqueness of solution of the following problem:

Given $f \in \mathcal{C}(F)$ find $u \in \mathcal{C}(F)$ such that $\mathcal{L}_{q}(u)=f$ on $F$.

Clearly, $[\mathrm{BVP}]$ or equivalently $[\mathrm{P}]$, is a self-adjoint problem since

$$
\int_{F} v \mathcal{L}_{q}(u) d x=\int_{F} u \mathcal{L}_{q}(v) d x \quad \text { for all } u, v \in \mathcal{C}(F)
$$

When $q \in \mathcal{C}^{+}(V)$ these boundary value problems have been extensively treated, see for instance $[3,4,10,15,20]$. Moreover, the authors have proved in [4] that the analysis of self-adjoint boundary value problems with general boundary conditions, namely Neumann, Robin or mixed boundary conditions, can be reduced to the study of a boundary value problem like [BVP] on a suitable network. For this reason, although in this paper we only consider Dirichlet problems and Poisson equations, all the results that will be obtained here concerning Schrödinger operators are in force for general boundary conditions.

To begin with, if $\sigma \in \mathcal{C}^{*}(V)$, then for each $u \in \mathcal{C}(V)$ and for each $x, y \in V$ it is verified that

$$
\sigma(x)(u(x)-u(y))=\sigma(x) \sigma(y)\left(\frac{u(x)}{\sigma(x)}-\frac{u(y)}{\sigma(y)}\right)+(\sigma(x)-\sigma(y)) u(x) .
$$

Therefore, considering the function $q_{\sigma}=-\frac{1}{\sigma} \mathcal{L}(\sigma)$, we obtain

$$
\mathcal{L}_{q}(u)(x)=\frac{1}{\sigma(x)} \int_{V} c(x, y) \sigma(x) \sigma(y)\left(\frac{u(x)}{\sigma(x)}-\frac{u(y)}{\sigma(y)}\right) d y+\left(q(x)-q_{\sigma}(x)\right) u(x),
$$

for each $u \in \mathcal{C}(V)$ and for each $x \in V$. As we will see in the following section, from this expression it follows easily that $\mathcal{L}_{q}$ is positive semidefinite when $q \geq q_{\sigma}$ for some $\sigma \in \mathcal{C}^{*}(V)$. This condition turns into $q \in \mathcal{C}^{+}(V)$ when $\sigma$ is constant. Before we go on, let us show some properties of the $q_{\sigma}$-type functions. 
Lemma 2.1 If $\sigma, \mu \in \mathcal{C}^{*}(V)$ then $q_{\sigma} \geq q_{\mu}$ iff $q_{\sigma}=q_{\mu}$ and this occurs iff $\sigma=a \mu$, for some $a>0$.

Proof. The first claim follows straightforwardly from the identities

$$
\int_{V}\left(q_{\sigma}-q_{\mu}\right) \sigma \mu d x=\int_{V}(\sigma \mathcal{L}(\mu)-\mu \mathcal{L}(\sigma)) d x=0 .
$$

Moreover, it is obvious that $q_{\sigma}=q_{\mu}$ when $\sigma=a \mu, a>0$. Conversely, if $q_{\sigma}=q_{\mu}$ then for all $x \in V$ we get that

$$
\begin{aligned}
0=\sigma(x) \mu(x)\left(q_{\sigma}(x)-q_{\mu}(x)\right) & =\int_{V} c(x, y)(\mu(x) \sigma(y)-\sigma(x) \mu(y)) d y \\
& =\int_{V} c(x, y) \mu(x) \mu(y)\left(\frac{\sigma(y)}{\mu(y)}-\frac{\sigma(x)}{\mu(x)}\right) d y
\end{aligned}
$$

If we consider the network $\bar{\Gamma}=(V, E, \bar{c})$, where $\bar{c}(x, y)=c(x, y) \mu(x) \mu(y)$, then $\bar{\Gamma}$ is connected and if $\overline{\mathcal{L}}$ denotes its associated combinatorial Laplacian, then $\overline{\mathcal{L}}\left(\frac{\sigma}{\mu}\right)=0$ and hence $\sigma=a \mu, a>0$.

The above result establishes that the function $q_{\sigma}$ determines $\sigma \in \mathcal{C}^{*}(V)$ up to multiplicative constant. In the most part of the paper, this lack of uniqueness will not be relevant. However, when it be suitable we will determine uniquely $\sigma$ from $q_{\sigma}$ throughout a normalization criterium. On the other hand, we also conclude that when $q_{\sigma} \neq q_{\mu}, q_{\sigma}$ determines a family of functions $q$ for which $\mathcal{L}_{q}$ is positive semidefinite that is essentially different of the family determined by $q_{\mu}$.

When $\sigma$ is not a constant function, $q_{\sigma}$ takes necessarily negative and positive values, since

$$
\int_{V} \sigma q_{\sigma} d x=-\int_{V} \mathcal{L}(\sigma) d x=0 .
$$

Therefore, the condition $q \geq q_{\sigma}$ allows $q$ to take negative values keeping the semipositive definiteness of $\mathcal{L}_{q}$. Next, we show throughout examples some aspects about the behavior of function $q_{\sigma}$. Firstly, we get upper and lower bounds for such a function. Since for each $x \in V$,

$$
q_{\sigma}(x)=\frac{1}{\sigma(x)} \int_{V} c(x, y)(\sigma(y)-\sigma(x)) d y
$$

if $\sigma_{m}=\min _{x \in V}\{\sigma(x)\}$ and $\sigma_{M}=\max _{x \in V}\{\sigma(x)\}$, then

$$
k(x)\left(\frac{\sigma_{m}}{\sigma_{M}}-1\right) \leq q_{\sigma}(x) \leq k(x)\left(\frac{\sigma_{M}}{\sigma_{m}}-1\right), \quad x \in V .
$$

In particular, this implies that $q_{\sigma}(x)>-k(x)$, for all $x \in V$. 
The function $\sigma$ can be chosen in such a way that $q_{\sigma}$ be a rapidly oscillating function. Specifically, consider a sequence of proper subsets of $V,\left\{F_{i}\right\}_{i=1}^{m}$, such that $\bar{F}_{i} \subset F_{i+1}$ for all $i=1, \ldots, m-1$ and take $F_{0}=\emptyset$ and $F_{m+1}=V$. Fixed $t \in(0,1)$, we define $\sigma_{t}(x)=1$ if $x \in F_{i}$ where $i$ is odd and $\sigma_{t}(x)=t$, if $x \in F_{i}$ when $i$ is even. Then, for all $x \in F_{i}$,

$$
q_{\sigma_{t}}(x)=a_{i} \int_{F_{i-1} \cup F_{i+1}} c(x, y) d y,
$$

where $a_{i}=(t-1)$, if $i$ is odd and $a_{i}=\left(\frac{1}{t}-1\right)$, if $i$ is even. In particular when $m=1$, letting $F=F_{1}$, we obtain $q_{\sigma_{t}}(x)<0$ if $x \in \delta\left(F^{c}\right), q_{\sigma_{t}}(x)>0$ if $x \in \delta(F)$ and $q_{\sigma_{t}}(x)=0$, otherwise.

Next we also show that for any proper subset $F \subset V$, there exists $\sigma \in$ $\mathcal{C}^{*}(V)$ such that $q_{\sigma}(x)<0$ for all $x \in F$. The key tool is the equilibrium measure for $F$, with respect to the kernel $\mathcal{L}$. Specifically, let $\nu^{F} \in \mathcal{C}^{*}(F)$ be the unique measure such that $\mathcal{L} \nu^{F}=1$ in $F$. The existence of such a measure was proved in [4], but it can be also deduced from the results we will obtain throughout this paper. Letting

$$
C_{F}=\max _{x \in \delta\left(F^{c}\right)} \int_{\delta(F)} c(x, y) d y,
$$

we define $\sigma=\nu^{F}+a 1_{F^{c}}$ where $0<a<C_{F}^{-1}$. Then,

$$
q_{\sigma}(x)= \begin{cases}-\frac{1}{\nu^{F}(x)}, & \text { if } x \in \stackrel{\circ}{F}, \\ -\frac{1}{\nu^{F}(x)}\left(1-a \int_{\delta(F)} c(x, y) d y\right), & \text { if } x \in \delta\left(F^{c}\right), \\ -\frac{1}{a} \int_{\delta\left(F^{c}\right)} c(x, y)\left(a-\nu^{F}(y)\right) d y, & \text { if } x \in \delta(F), \\ 0, & \text { if } x \in \operatorname{Ext}(F) .\end{cases}
$$

So, $q_{\sigma}(x)<0$ for all $x \in F$. To end this section, we build a function of this type for a subset $F$ of a distance-regular graph of degree $k$, such that $|F|=n-1$. Fixed $y \in V$, let $F=V \backslash\{y\}$. In this case,

$$
\nu^{F}(x)=\sum_{j=0}^{d(x, y)-1} \frac{n-\left|B_{j}\right|}{\left|\partial B_{j}\right|}, \quad x \in V,
$$

where $B_{j}=\{z \in V: d(z, y) \leq j\}$ and $\partial B_{j}=\left\{(x, y) \in E: x \in B_{j}^{c}, y \in B_{j}\right\}$, $($ see $[4]$. 
Moreover, $C_{F}=1$ and therefore if $0<a<1$ then

$$
q_{\sigma}(x)= \begin{cases}-\left(\sum_{j=0}^{d(x, y)-1} \frac{n-\left|B_{j}\right|}{\left|\partial B_{j}\right|}\right)^{-1}, & \text { if } d(x, y) \geq 2, \\ -\frac{k}{n-1}(1-a), & \text { if } d(x, y)=1 \\ \frac{n-1}{a}-k, & \text { if } x=y\end{cases}
$$

\section{Bilinear forms associated to Schrödinger operators}

In this section we start obtaining the expression of the bilinear form, $\mathcal{E}_{q}$, associated with the Schrödinger operator $\mathcal{L}_{q}$. Clearly, for $u, v \in \mathcal{C}(V)$ we get that

$$
\begin{aligned}
\mathcal{E}_{q}(u, v)=\frac{1}{2} & \int_{V} \int_{V} c(x, y)(u(x)-u(y))(v(x)-v(y)) d x d y \\
& +\int_{V} q(x) u(x) v(x) d x .
\end{aligned}
$$

It is well-known that when $q \in \mathcal{C}^{+}(V)$, the quadratic form associated with $\mathcal{E}_{q}$ satisfies important properties. Our aim in this section is to study both the properties that are in force and the new ones when we eliminate the constraint on $q$ of being non negative. So, to compare the general case with the non negative one, it will be suitable to summarize the known properties for the case in which the operator $\mathcal{L}_{q}$ has associated a Dirichlet form, see $[1,2,8,14]$.

A transformation $T: \mathcal{C}(V) \rightarrow \mathcal{C}(V)$ is called contraction if for all $u \in \mathcal{C}(V)$,

$$
|T(u)(x)-T(u)(y)| \leq|u(x)-u(y)| \text { for all } x, y \in V .
$$

Moreover, a contraction $T$ is called normal contraction if $|T(u)| \leq|u|$, for all $u \in \mathcal{C}(V)$. The three fundamental normal contractions are:

a) The null contraction, $T(u)=0$, for all $u \in \mathcal{C}(V)$.

b) The modulus contraction, $T(u)=|u|$, for all $u \in \mathcal{C}(V)$.

c) The unit contraction, $T(u)=u^{+} \wedge 1$, for all $u \in \mathcal{C}(V)$.

If $\mathcal{E}: \mathcal{C}(V) \times \mathcal{C}(V) \longrightarrow \mathbb{R}$ is a symmetric bilinear form we say that a transformation $T: \mathcal{C}(V) \longrightarrow \mathcal{C}(V)$ operates with respect to $\mathcal{E}$ if $\mathcal{E}(T(u), T(u)) \leq$ $\mathcal{E}(u, u)$, for all $u \in \mathcal{C}(V)$. Clearly, the null contraction operates w.r.t. $\mathcal{E}$ iff $\mathcal{E}$ is positive semidefinite and the modulus contraction operates w.r.t. $\mathcal{E}$ iff $\mathcal{E}\left(\varepsilon_{x}, \varepsilon_{y}\right) \leq 0$, for all $x, y \in V, x \neq y$. 
A symmetric bilinear form $\mathcal{E}: \mathcal{C}(V) \times \mathcal{C}(V) \longrightarrow \mathbb{R}$ is called a Dirichlet form if the unit contraction operates w.r.t. $\mathcal{E}$, that is if $\mathcal{E}\left(u^{+} \wedge 1, u^{+} \wedge 1\right) \leq$ $\mathcal{E}(u, u)$, for all $u \in \mathcal{C}(V)$. The following result is well-known, see [1].

Proposition 3.1 A symmetric bilinear form $\mathcal{E}$ is a Dirichlet form iff any normal contraction operates w.r.t. $\mathcal{E}$. Moreover, this condition is equivalent to the following ones:

$$
\begin{aligned}
\mathcal{E}\left(\varepsilon_{x}, \varepsilon_{y}\right) & \leq 0, \quad \text { for all } x, y \in V, x \neq y \quad \text { and } \\
\int_{V} \mathcal{E}\left(\varepsilon_{z}, \varepsilon_{y}\right) d y & \geq 0, \quad \text { for all } z \in V .
\end{aligned}
$$

As $\mathcal{E}_{q}\left(\varepsilon_{x}, \varepsilon_{y}\right)=-c(x, y)$, for all $x, y \in V, x \neq y$, and $q(x)=\int_{V} \mathcal{E}_{q}\left(\varepsilon_{x}, \varepsilon_{y}\right) d y$, for all $x \in V$, from the above proposition it follows that $\mathcal{E}_{q}$ is a Dirichlet form iff $q \in \mathcal{C}^{+}(V)$.

Next we tackle the study of $\mathcal{E}_{q}$ when it is not necessarily a Dirichlet form. Since for arbitrary $q \in \mathcal{C}(V)$ the modulus contraction operates w.r.t. $\mathcal{E}_{q}$, we are interested in identifying those $q \in \mathcal{C}(V)$ for which the null contraction operates w.r.t. $\mathcal{E}_{q}$. Firstly, note that if $\sigma \in \mathcal{C}^{*}(V)$, by identity (2.1), the bilinear form $\mathcal{E}_{q}$ can be re-written as

$$
\begin{aligned}
\mathcal{E}_{q}(u, v) & =\frac{1}{2} \int_{V} \int_{V} c(x, y) \sigma(x) \sigma(y)\left(\frac{u(x)}{\sigma(x)}-\frac{u(y)}{\sigma(y)}\right)\left(\frac{v(x)}{\sigma(x)}-\frac{v(y)}{\sigma(y)}\right) d x d y \\
& +\int_{V}\left(q(x)-q_{\sigma}(x)\right) u(x) v(x) d x .
\end{aligned}
$$

So, $\mathcal{E}_{q}$ is positive semidefinite when $q \geq q_{\sigma}$. Moreover, the above expression motivates the following concepts.

Definition 3.2 If $\sigma \in \mathcal{C}^{*}(V)$, we say that $T: \mathcal{C}(V) \longrightarrow \mathcal{C}(V)$ is a $\sigma$-contraction if for all $u \in \mathcal{C}(V)$,

$$
\left|\frac{T(u)(x)}{\sigma(x)}-\frac{T(u)(y)}{\sigma(y)}\right| \leq\left|\frac{u(x)}{\sigma(x)}-\frac{u(y)}{\sigma(y)}\right|, \text { for all } x, y \in V .
$$

A $\sigma$-contraction $T$ is called normal $\sigma$-contraction if $|T(u)| \leq|u|$, for all $u \in \mathcal{C}(V)$.

Note that the null and the modulus contractions are normal $\sigma$-contractions for all $\sigma \in \mathcal{C}^{*}(V)$. Moreover, when $\sigma$ is constant then the $\sigma$-contractions are exactly the contractions. More generally, $T$ is a $\sigma$-contraction (normal $\sigma$-contraction) iff there exists $\hat{T}$ a contraction (normal contraction) such that $T(u)=\sigma \hat{T}\left(\frac{u}{\sigma}\right)$, for all $u \in \mathcal{C}(V)$. 
The properties of the quadratic forms associated with Schrödinger operators are gathered together in the following result.

Proposition 3.3 Let $\mathcal{E}_{q}$ be the bilinear form associated with the Schrödinger operator $\mathcal{L}_{q}$. Then the following statements are equivalent:

(i) The bilinear form $\mathcal{E}_{q}$ is positive semidefinite.

(ii) There exist $\sigma \in \mathcal{C}^{*}(V)$ and $a \geq 0$ such that $q=q_{\sigma}+a 1$.

(iii) There exists $\sigma \in \mathcal{C}^{*}(V)$ such that $q \geq q_{\sigma}$.

(iv) There exists $\sigma \in \mathcal{C}^{*}(V)$ such that any normal $\sigma$-contraction operates w.r.t. $\mathcal{E}_{q}$.

(v) There exists $\sigma \in \mathcal{C}^{*}(V)$ such that $\mathcal{E}_{q}\left(u^{+} \wedge \sigma, u^{+} \wedge \sigma\right) \leq \mathcal{E}_{q}(u, u)$, for any $u \in \mathcal{C}(V)$.

Proof. If $\mathcal{E}_{q}$ is positive semidefinite, then the matrix associated with $\mathcal{E}_{q}$, is a symmetric positive semidefinite matrix with non positive off-diagonal entries, i.e., a symmetric M-matrix. In addition, it is irreducible since $\Gamma$ is connected. Then, its lowest eigenvalue, $a$, is non negative and has an eigenvector, $\sigma$, whose entries are strictly positive, (see [6, Th. 4.16]). So,

$$
\mathcal{L}(\sigma)+q \sigma=a \sigma
$$

which implies that $q=q_{\sigma}+a 1$.

Clearly, (ii) implies (iii) and (iii) implies (iv) by expression (3.2). Moreover, (iv) implies (v), since $T(u)=u^{+} \wedge \sigma$ is a normal $\sigma$-contraction.

At last, if (v) holds then to prove (i) it is enough to show that $q \geq q_{\sigma}$. So, for each $x \in V$ and $t>0$ consider $u_{t}=\sigma+t \varepsilon_{x}$. Then, $u_{t}^{+} \wedge \sigma=\sigma$ which implies that $0 \leq 2 \mathcal{E}_{q}\left(\sigma, \varepsilon_{x}\right)+t \mathcal{E}_{q}\left(\varepsilon_{x}, \varepsilon_{x}\right)$ for all $t>0$ and hence $0 \leq$ $\mathcal{E}_{q}\left(\sigma, \varepsilon_{x}\right)=\left(q(x)-q_{\sigma}(x)\right) \sigma(x)$.

Observe that if $q$ is such that $\mathcal{E}_{q}$ is positive semidefinite then the function $q_{\sigma}$ and the value $a$ obtained in part (ii) of the above proposition are uniquely determined, since if $q_{\sigma}+a=q_{\mu}+b$ and $a \geq b$, then $q_{\mu}-q_{\sigma} \geq 0$ which implies, by Lemma 2.1, $q_{\mu}=q_{\sigma}$ and hence $a=b$. Therefore, if we define

$$
\mathcal{C}_{n}^{*}(V)=\left\{\sigma \in \mathcal{C}^{*}(V): \frac{1}{n} \int_{V} \sigma d x=1\right\}
$$

then the set

$$
\bigcup_{\sigma \in \mathcal{C}_{n}^{*}(V)}\left\{q_{\sigma}+a: a \geq 0\right\}
$$

is a partition of the set of functions $q$ such that $\mathcal{E}_{q}$ is positive semidefinite. 
Corollary 3.4 Let $\mathcal{E}_{q}$ be the bilinear form associated with the Schrödinger operator $\mathcal{L}_{q}$. Then the following statements are equivalent:

(i) There exists $\sigma \in \mathcal{C}^{*}(V)$ such that any $\sigma$-contraction operates w.r.t. $\mathcal{E}_{q}$.

(ii) The bilinear form $\mathcal{E}_{q}$ is positive semidefinite but not strictly definite.

(iii) There exists $\sigma \in \mathcal{C}^{*}(V)$ such that $q=q_{\sigma}$.

Proof. If (i) is satisfied, $\mathcal{E}_{q}$ is positive semidefinite and hence

$$
\mathcal{E}_{q}(\sigma, \sigma) \geq 0
$$

since any normal $\sigma$-contraction is a $\sigma$-contraction by Proposition 3.3. On the other hand, since the transformation $T(u)=u+\sigma$ is a $\sigma$-contraction,

$$
4 \mathcal{E}_{q}(\sigma, \sigma)=\mathcal{E}_{q}(T(\sigma), T(\sigma)) \leq \mathcal{E}_{q}(\sigma, \sigma)
$$

which implies that $\mathcal{E}_{q}(\sigma, \sigma)=0$.

If (ii) is satisfied, then by Proposition 3.3, there exists $\sigma \in \mathcal{C}^{*}(V)$ such that $q \geq q_{\sigma}$. In addition, since $\mathcal{E}_{q}$ is not strictly definite there exists non-zero $u \in \mathcal{C}(V)$ such that $\mathcal{E}_{q}(u, u)=0$ and then

$0=\frac{1}{2} \int_{V} \int_{V} c(x, y) \sigma(x) \sigma(y)\left(\frac{u(x)}{\sigma(x)}-\frac{u(y)}{\sigma(y)}\right)^{2} d x d y=\int_{V}\left(q(x)-q_{\sigma}(x)\right) u(x)^{2} d x$.

Since $\Gamma$ is connected, the first equality implies that $u$ is a non-zero multiple of $\sigma$ and therefore $q=q_{\sigma}$, from the second equality.

Finally, it is clear that (iii) implies (i).

From the expression (3.2) it is clear that, if for each $\sigma \in \mathcal{C}^{*}(V)$ we define the network $\hat{\Gamma}=(V, E, c \cdot \sigma \otimes \sigma)$ then $\mathcal{E}_{q}(u, v)=\hat{\mathcal{E}}\left(\frac{u}{\sigma}, \frac{v}{\sigma}\right)$, where $\hat{\mathcal{E}}$ is the bilinear form associated with the Schrödinger operator $\hat{\mathcal{L}}+\left(q-q_{\sigma}\right)$ and where $\hat{\mathcal{L}}$ is the combinatorial Laplacian of $\hat{\Gamma}$. The above results show that $\mathcal{E}_{q}$ is a positive semidefinite form iff there exists $\sigma \in \mathcal{C}^{*}(V)$ such that $\hat{\mathcal{E}}$ is a Dirichlet form.

We conclude this section observing that we have proved that the Schrödinger operator $\mathcal{L}_{q}$ is positive semidefinite iff there exists $\sigma \in \mathcal{C}^{*}(V)$ such that $q \geq q_{\sigma}$. In addition if $q \neq q_{\sigma}$, then $\mathcal{L}_{q}$ is invertible, whereas if $q=q_{\sigma}$ then $\mathcal{L}_{q}$ is singular. In this case, the eigenvalue 0 is simple and its eigenvector subspace is generated by $\sigma$. These properties allow to obtain the following results about the existence and uniqueness of solution for [BVP] and about its variational formulation. 
Proposition 3.5 (Dirichlet principle) Let $F$ be a non empty subset of $V$, and suppose that there exists $\sigma \in \mathcal{C}^{*}(V)$ such that $q \geq q_{\sigma}$. Given $f \in \mathcal{C}(F)$ and $g \in \mathcal{C}(\delta(F))$, consider the convex set $C_{g}=\{v \in \mathcal{C}(\bar{F}): v=g$ on $\delta(F)\}$ and the quadratic functional $\mathcal{J}_{q}: \mathcal{C}(V) \longrightarrow \mathbb{R}$ determined by the expresion

$\mathcal{J}_{q}(u)=\frac{1}{2} \int_{\bar{F}} \int_{\bar{F}} c(x, y)(u(x)-u(y))^{2} d x d y+\int_{\bar{F}} q(x) u(x)^{2} d x-2 \int_{F} f(x) u(x) d x$.

Then $u \in \mathcal{C}(\bar{F})$ is a solution of $[\mathrm{BVP}]$ iff $u$ minimizes $\mathcal{J}_{q}$ on $C_{g}$. Moreover, if it is not simultaneously true that $F=V$ and $q=q_{\sigma}$, then $\mathcal{J}_{q}$ has a unique minimum on $C_{g}$. Otherwise, $\mathcal{J}_{q}$ has a minimum iff $\int_{V} f(x) \sigma(x) d x=0$. In this case, there exists a unique minimum $u \in \mathcal{C}(V)$ such that $\int_{V} u(x) \sigma(x) d x=0$.

Proof. Observe first that $C_{g}=g+\mathcal{C}(F)$ and that as

$$
\mathcal{J}_{q}(v)=\mathcal{E}_{q}(v, v)-2 \int_{F} f v d x, \quad \text { for all } v \in \mathcal{C}(F),
$$

then by Proposition 3.3, $\mathcal{J}_{q}$ is a convex functional on $\mathcal{C}(F)$ and hence on $C_{g}$. Moreover, by Corollary 3.4, it is an strictly convex functional iff it is not simultaneously true that $F=V$ and $q=q_{\sigma}$ and then $\mathcal{J}_{q}$ has a unique minimum on $C_{g}$.

On the other hand, when $F=V$ and $q=q_{\sigma}$ simultaneously the minima of $\mathcal{J}_{q}$ are characterized by the Euler identity:

$$
\mathcal{E}_{q}(u, v)=\int_{V} f v d x, \quad \text { for all } v \in \mathcal{C}(V) .
$$

Since in this case $\mathcal{E}_{q}(u, \sigma)=0$ for all $u \in \mathcal{C}(V)$, necessarily $f$ must satisfy that $\int_{V} f \sigma d x=0$. Moreover if this condition holds and $\mathcal{V}$ denotes the vectorial subspace generated by $\sigma$, then $u \in \mathcal{V}^{\perp}$ minimizes $\mathcal{J}_{q}$ on $\mathcal{V}^{\perp}$ iff $\mathrm{u}$ minimizes $\mathcal{J}_{q}$ on $\mathcal{C}(V)$ and the existence of minimum follows since $\mathcal{J}_{q}$ is strictly convex on $\mathcal{V}^{\perp}$.

In any case, the equation described in $[\mathrm{BVP}]$ is the Euler-Lagrange identity for the corresponding minimization problem.

The above proposition establishes that when $q=q_{\sigma}$ the Poisson equation $\mathcal{L}_{q}(u)=f$ has a solution iff $\int_{V} f \sigma d x=0$. Observe that under this condition if $v$ is a solution, then the set of solutions is given by $\{v+a \sigma: a \in \mathbb{R}\}$ and hence

$$
u=v-\frac{\sigma}{\|\sigma\|_{2}^{2}} \int_{V} v \sigma d x
$$

is the unique solution such that $\int_{V} u \sigma d x=0$. 


\section{Monotonicity of the Schrödinger operators}

It is well-known that when $q \geq 0$, the Schrödinger operator $\mathcal{L}_{q}$, satisfies the weak minimum principle which is equivalent to the monotonicity property, see for instance $[19,20]$. Essentially, this is the discrete analogous of the weak minimum principle for second order elliptic operators (see [13].) In this section, we start by showing the monotonicity property of a Schrödinger operator when its associated bilinear form is positive semidefinite. We will also deduce some relevant consequences of this fact. Unlike the continuous case, in the discrete framework there is not difference between classical and weak solutions of Dirichlet problems. Therefore, we will prove a discrete analogue of the Hopf's minimum principle. To our knowledge, up to now this property has not been analyzed in the discrete case, even in the case $q \geq 0$. An important consequence of Hopf's minimum principle is a generalization of the condenser problem for the Schrödinger operator $\mathcal{L}_{q}$. In addition, the solution of this problem allows us to extend the concept of effective resistance between two subsets of $V$.

If it is not mentioned otherwise, in the rest of this section we will assume the following hypotheses:

H1: There exists $\sigma \in \mathcal{C}^{*}(V)$ such that $q \geq q_{\sigma}$.

H2: It is not simultaneously true that $F=V$ and $q=q_{\sigma}$, that is $F$ is any non empty subset of $V$ except when $q=q_{\sigma}$ in which case $F$ is a proper subset.

The following result establishes the monotonicity of the Schrödinger operators, under the above-mentioned hypotheses.

Proposition 4.1 If $u \in \mathcal{C}(V)$ is such that $\mathcal{L}_{q}(u) \geq 0$ on $F$ and $u \geq 0$ on $F^{c}$, then $u \in \mathcal{C}^{+}(V)$.

Proof. If we denote $v=u / \sigma$, then to conclude it is enough to prove that $v \in$ $\mathcal{C}^{+}(F)$. Indeed, if $x \in F$ is such that $v(x)=\min _{z \in F}\{v(z)\}$ it suffices to prove that $v(x) \geq 0$, or equivalently that if $v(x) \leq 0$ then necessarily $v(x)=0$.

Suppose that $v(x) \leq 0$. Then $v(x) \leq v(y)$ for all $y \in V$ and therefore, from expression (2.1) we deduce that

$$
\begin{aligned}
& 0 \leq \mathcal{L}_{q}(u)(x)= \\
& =\frac{1}{\sigma(x)} \int_{V} c(x, y) \sigma(x) \sigma(y)(v(x)-v(y)) d y+\left(q(x)-q_{\sigma}(x)\right) \sigma(x) v(x) \leq 0,
\end{aligned}
$$

which implies that $v(x)=v(y)$ for all $y \in V$ such that $x \sim y$. Hence $v=a 1$, with $a \in \mathbb{R}$, since $\Gamma$ is connected.

When $F \neq V$, necessarily $v(x)=0$, since $v \geq 0$ on $F^{c}$, whereas if $F=V$, as $q \neq q_{\sigma}$ and $0=\mathcal{L}_{q}(u)=a\left(q-q_{\sigma}\right) \sigma$, then $a=0$. 
Observe that when $q=q_{\sigma}$, then

$$
\int_{V} \sigma(x) \mathcal{L}_{q}(u)(x) d x=0, \quad \text { for all } u \in \mathcal{C}(V) .
$$

Therefore, if $u \in \mathcal{C}(V)$ is such that $\mathcal{L}_{q}(u) \geq 0$, then $\mathcal{L}_{q}(u)=0$ and $u=a \sigma$, $a \in \mathbb{R}$. This result can be obtained by reasoning as in the above proposition.

Corollary 4.2 Let $p \in \mathcal{C}(V)$ be such that $p \geq q$. If $u, v \in \mathcal{C}(V)$ satisfy that $\mathcal{L}_{q}(u) \geq \mathcal{L}_{p}(v) \geq 0$ on $F$ and $u \geq v \geq 0$ on $F^{c}$, then $u \geq v \geq 0$ on $V$.

Proof. By the above proposition, we have that $v \in \mathcal{C}^{+}(V)$. On the other hand, since $\mathcal{L}_{q}=\mathcal{L}_{p}-(p-q)$, the function $w=u-v$ satisfies that $\mathcal{L}_{q}(w)=\mathcal{L}_{q}(u)-\mathcal{L}_{p}(v)+(p-q) v \geq 0$ on $F$ and $w \geq 0$ on $F^{c}$. So, the result follows from the above proposition.

Now, we obtain a new proof of the existence and uniqueness of solution for problem $[\mathrm{P}]$, which includes a property of the support of the solution.

Corollary 4.3 For each $f \in \mathcal{C}(F)$ there exists a unique $u \in \mathcal{C}(F)$ such that $\mathcal{L}_{q}(u)=f$ on $F$. In addition, if $f \in \mathcal{C}^{+}(F)$ then $u \in \mathcal{C}^{+}(F)$ and $\operatorname{supp}(f) \subset \operatorname{supp}(u)$.

Proof. Consider the endomorphism $\mathcal{F}: \mathcal{C}(F) \longrightarrow \mathcal{C}(F)$ given by $\mathcal{F}(u)=$ $\mathcal{L}_{q}(u)_{\left.\right|_{F}}$. By Proposition $4.1, \mathcal{F}$ is monotone which implies that it is an isomorphism and that $u \in \mathcal{C}^{+}(F)$ when $f \in \mathcal{C}^{+}(F)$. Moreover if $u(x)=0$, then

$$
f(x)=\mathcal{L}_{q}(u)(x)=-\int_{V} c(x, y) u(y) d y \leq 0
$$

and hence $f(x)=0$.

The monotonicity property of the operator $\mathcal{L}_{q}$ showed in Proposition 4.1, can be interpreted as, and in fact is equivalent to, a weak minimum principle for the difference operator $\mathcal{L}_{q}$. We next show that such property is in fact a strong minimum principle. Before describing the results we must point out that, unlike the continuous case, in the discrete setting concepts such as interior, boundary and closure of a set have geometrical nature. So, we can consider two types of boundaries associated with a set $F$, its vertex or exterior boundary $\delta(F)$ and its interior boundary, $\delta\left(F^{c}\right)$. Both boundaries have been considered in the literature, depending on which set plays the role of open set. The first case correspond to consider $F$ as an open set, see for instance $[4,9]$, whereas in the second one this role is played by $\stackrel{\circ}{F}$, see for instance $[12,15]$. The Second Green Identity and the fact that $F \cap \delta(F)=\emptyset$ whereas $\stackrel{\circ}{F} \cup \delta(\stackrel{\circ}{F}) \neq F$ in general, are some of the reasons why we prefer the exterior boundary formulation. However, in the following results both types of boundaries will be considered. 
Proposition 4.4 (Minimum principle) Suppose that $q=q_{\sigma}$ and let $F$ be a proper connected subset. If $u \in \mathcal{C}(\bar{F})$ is such that $\mathcal{L}_{q}(u) \geq 0$ on $F$, then

$$
\min _{x \in \delta(F)}\left\{\frac{u(x)}{\sigma(x)}\right\} \leq \min _{x \in \delta\left(F^{c}\right)}\left\{\frac{u(x)}{\sigma(x)}\right\} \leq \min _{x \in F}\left\{\frac{u(x)}{\sigma(x)}\right\} .
$$

Moreover, the first inequality is an equality iff $u$ coincides on $\bar{F}$ with a multiple of $\sigma$.

Proof. To prove the first inequality, let

$$
m=\min _{x \in \delta(F)}\left\{\frac{u(x)}{\sigma(x)}\right\}
$$

and consider $w=u-m \sigma_{\left.\right|_{\bar{F}}}$. Of course, $\mathcal{L}_{q}(w)=\mathcal{L}_{q}(u) \geq 0$ on $F$ and $w \geq 0$ on $F^{c}$, which implies $w \geq 0$ on $V$, by Proposition 4.1. Therefore,

$$
m=\min _{x \in \bar{F}}\left\{\frac{u(x)}{\sigma(x)}\right\} .
$$

Repeating the same argument for the set $H=\stackrel{\circ}{F}$ and for the function $u_{\left.\right|_{\bar{H}}}$ and keeping in mind that $F=\delta\left(F^{c}\right) \cup \stackrel{\circ}{F}$ and that $\delta(\stackrel{\circ}{F}) \subset \delta\left(F^{c}\right)$ we get the second inequality.

On the other hand, if we consider

$$
v=\frac{w}{\sigma}=\frac{u}{\sigma}-m
$$

then $v \geq 0$ on $\bar{F}$. Moreover, if $x^{*} \in F$ is such that $m=\frac{u\left(x^{*}\right)}{\sigma\left(x^{*}\right)}$, it follows $v\left(x^{*}\right)=0$. Reasoning as in Proposition 4.1, we get $v(z)=0$ for each $z \in \bar{F}$ such that $z \sim x^{*}$. Iterating this argument, we conclude that $v=0$ on $\bar{F}$, since $\bar{F}$ is connected.

Of course an analogous result is satisfied for the maximum values of functions $u \in \mathcal{C}(V)$ such that $\mathcal{L}_{q}(u) \leq 0$ on $F$. The following result combines both cases and it is a discrete analogue of the maximum and minimum principles for functions such that $\mathcal{L}_{q}(u)=0$.

Corollary 4.5 Suppose that $q=q_{\sigma}$ and let $F$ be a proper connected subset. If $u \in \mathcal{C}(\bar{F})$ is such that $\mathcal{L}_{q}(u)=0$ on $F$, then the following properties hold:

(i) For all $x \in F, \min _{z \in \delta(F)}\left\{\frac{u(z)}{\sigma(z)}\right\} \leq \frac{u(x)}{\sigma(x)} \leq \max _{z \in \delta(F)}\left\{\frac{u(z)}{\sigma(z)}\right\}$ and either of the two inequalities is an equality iff $u$ coincides on $\bar{F}$ with a multiple of $\sigma$.

(ii) For all $x \in \stackrel{\circ}{F}, \min _{z \in \delta\left(F^{c}\right)}\left\{\frac{u(z)}{\sigma(z)}\right\} \leq \frac{u(x)}{\sigma(x)} \leq \max _{z \in \delta\left(F^{c}\right)}\left\{\frac{u(z)}{\sigma(z)}\right\}$. 
We must observe that the minimum principle for discrete Schrödinger operators, studied in the above proposition, is sharper than its continuous analogue, since in the continuous case the 0 -order term must vanish whereas in the discrete setting the condition is $q=q_{\sigma}$. In particular, when $F \neq V$ we know that there exists $\sigma \in \mathcal{C}^{*}(V)$ such that $q_{\sigma}<0$ on $F$. So, the minimum principle in the discrete case can be verified for Schrödinger operators with suitable negative 0 -order terms.

Now, we prove the minimum principle when $q \geq q_{\sigma}$. We remark that although the results are analogous to those of the continuous case, we can not employ the same techniques that in [13], because they are based on continuity arguments.

Proposition 4.6 (Hopf's minimum principle) Consider $F$ a non empty connected subset and $u \in \mathcal{C}(\bar{F})$ such that $\mathcal{L}_{q}(u) \geq 0$ on $F$ and suppose that there exists $x^{*} \in F$ such that

$$
u\left(x^{*}\right) \leq 0 \quad \text { and } \quad \frac{u\left(x^{*}\right)}{\sigma\left(x^{*}\right)}=\min _{x \in \bar{F}}\left\{\frac{u(x)}{\sigma(x)}\right\} .
$$

Then $u$ coincides on $\bar{F}$ with a non positive multiple of $\sigma, \mathcal{L}_{q}(u)=0$ on $F$ and either $u=0$ or $q=q_{\sigma}$ on $F$.

Proof. Taking in mind the expression $(2.1)$ for $\mathcal{L}_{q}$, we have that

$$
\mathcal{L}_{q}(u)\left(x^{*}\right) \leq 0
$$

and therefore, $\mathcal{L}_{q}(u)\left(x^{*}\right)=0$. This implies that

$$
\frac{u(y)}{\sigma(y)}=\frac{u\left(x^{*}\right)}{\sigma\left(x^{*}\right)} \quad \text { for all } y \sim x^{*}
$$

and either $u\left(x^{*}\right)=0$ or $q\left(x^{*}\right)=q_{\sigma}\left(x^{*}\right)$. The result follows by reasoning as in Proposition 4.1.

Under the above conditions, when $F=V$, hypothesis $\mathrm{H} 2$ implies that $u=0$. On the other hand, by applying the Hopf's minimum principle to $-u$, it is easy to conclude the Hopf's maximum principle, that is if $u \in \mathcal{C}(\bar{F})$ is such that $\mathcal{L}_{q}(u) \leq 0$ on $F$, then the maximum value of $\frac{u}{\sigma}$ on $\bar{F}$ can not be attained at $F$ if it is positive, except when $u$ is a non negative multiple of $\sigma$.

The general minimum principle can be obtained as a consequence of Hopf's minimum principle. Before proving it, we show that the sets of supersolutions and subsolutions of the equation $\mathcal{L}_{q}(u)=0$ are closed by taking min and max respectively. 
Lemma 4.7 Given $u, v \in \mathcal{C}(V)$, consider the sets

$$
\begin{aligned}
& F_{1}=\left\{x \in V: \mathcal{L}_{q}(u)(x) \wedge \mathcal{L}_{q}(v)(x) \geq 0\right\} \quad \text { and } \\
& F_{2}=\left\{x \in V: \mathcal{L}_{q}(u)(x) \vee \mathcal{L}_{q}(v)(x) \leq 0\right\} .
\end{aligned}
$$

Then, $\mathcal{L}_{q}(u \wedge v)(x) \geq 0$ for all $x \in F_{1}$ and $\mathcal{L}_{q}(u \vee v)(x) \leq 0$ for all $x \in F_{2}$.

Proof. It suffices to note that when $u(x) \leq v(x)$, then

$$
\mathcal{L}_{q}(u \wedge v)(x) \geq \mathcal{L}_{q}(u)(x) \quad \text { and } \quad \mathcal{L}_{q}(u \vee v)(x) \leq \mathcal{L}_{q}(v)(x)
$$

Corollary 4.8 Let $F$ be a proper subset of $V, u \in \mathcal{C}(\bar{F})$ such that $\mathcal{L}_{q}(u) \geq 0$ on F. Then,

$$
\min _{\delta(F)}\left\{\frac{u \wedge 0}{\sigma}\right\} \leq \min _{F}\left\{\frac{u}{\sigma}\right\} \quad \text { and } \quad \min _{\delta\left(F^{c}\right)}\left\{\frac{u \wedge 0}{\sigma}\right\} \leq \min _{\stackrel{\circ}{F}}\left\{\frac{u}{\sigma}\right\}
$$

Proof. If $v=u \wedge 0$, then from the above lemma we get that $\mathcal{L}_{q}(v) \geq 0$ on $F$. Since $v \leq 0$ on $V$, by applying the Hopf's minimum principle to each connected component of $F$ we obtain that

$$
\min _{\delta(F)}\left\{\frac{v}{\sigma}\right\} \leq \min _{F}\left\{\frac{v}{\sigma}\right\} \leq \min _{F}\left\{\frac{u}{\sigma}\right\} \text { and } \min _{\delta\left(F^{c}\right)}\left\{\frac{v}{\sigma}\right\} \leq \min _{\stackrel{\circ}{F}}\left\{\frac{v}{\sigma}\right\} \leq \min _{\stackrel{\circ}{F}}\left\{\frac{u}{\sigma}\right\}
$$

where we have also used that $u \wedge 0 \leq u$.

Of course when $\mathcal{L}_{q}(u) \leq 0$, an analogous property holds for the maximum value of $u$ on $F$ and on $\stackrel{\circ}{F}$ replacing $u \wedge 0$ by $u^{+}$. Moreover, the result is more accurate for solutions of the equation $\mathcal{L}_{q}(u)=0$.

Corollary 4.9 Let $F$ be a proper subset of $V$ and $u \in \mathcal{C}(\bar{F})$ such that $\mathcal{L}_{q}(u)=0$ on $F$. Then,

$$
\max _{\substack{\circ \\ F}}\left\{\frac{|u|}{\sigma}\right\} \leq \max _{\delta\left(F^{c}\right)}\left\{\frac{|u|}{\sigma}\right\} \leq \max _{\delta(F)}\left\{\frac{|u|}{\sigma}\right\} .
$$

Proof. Since $|u|=-u \vee u$ and $u$ and $-u$ are both subsolutions, then applying Lemma 4.7 we get that $\mathcal{L}_{q}(|u|) \leq 0$ on $F$. Since $|u| \in \mathcal{C}^{+}(V)$, the result follows from Hopf's maximum principle.

In the following result we show that the minimum principle implies the monotonicity of $\mathcal{L}_{q}$. Moreover, we can also precise the last conclusion of Corollary 4.3. 
Proposition 4.10 Consider $u \in \mathcal{C}(\bar{F})$ such that $\mathcal{L}_{q}(u) \geq 0$ on $F$. If $u(x) \geq 0$ for all $x \in \delta(F)$, then $u \in \mathcal{C}^{+}(\bar{F})$. In addition, if $H$ is a connected component of $F$, either $u=0$ or $u>0$ on $H$.

Proof. The case $F=V$ follows straightforwardly. So, we suppose that $F$ is a proper subset. Applying Corollary 4.8, we get that $u \in \mathcal{C}^{+}(F)$ and therefore $u \in \mathcal{C}^{+}(\bar{F})$. Moreover when $H$ is a connected component of $F$, if $u\left(x^{*}\right)=0$ for some $x^{*} \in H$, then $u=0$ on $H$ by applying the Hopf's minimum principle to $\bar{H}$.

We conclude this section obtaining a generalization of the well-known Condenser principle, that in its classical version is closely related to the theory of Dirichlet Forms. The case when $\sigma$ is a constant function, that is when $q \in \mathcal{C}^{+}(V)$, was studied in [5].

Proposition 4.11 (Condenser principle) Let $F$ be a proper subset of $V$, $\{A, B\}$ a partition of $\delta(F)$ and $u \in \mathcal{C}(\bar{F})$ the unique solution of the following boundary value problem

$$
\left.\begin{array}{cl}
\mathcal{L}_{q}(u)(x)=0, & \text { if } x \in F, \\
u(x)=\sigma(x), & \text { if } x \in A, \\
u(x)=0, & \text { if } x \in B .
\end{array}\right\}
$$

Then, $0 \leq u \leq \sigma$ on $V, \mathcal{L}_{q}(u) \geq 0$ on $A$ and $\mathcal{L}_{q}(u) \leq 0$ on $B$. Moreover, if $H$ is a connected component of $F$, then $u>0$ on $H$ when $\delta(H) \cap A \neq \emptyset$, $u=0$ on $H$ when $\delta(H) \cap A=\emptyset, u<\sigma$ on $H$ when either $\delta(H) \cap B \neq \emptyset$ or $q \neq q_{\sigma}$ on $H$ and $u=\sigma$ on $H$ when $\delta(H) \cap B=\emptyset$ and $q=q_{\sigma}$ on $H$, simultaneously.

Proof. When $A=\emptyset$ necessarily $u=0$ since $F^{c} \neq \emptyset$, and then the result follows. Suppose now that $A \neq \emptyset$ and consider $f=-\mathcal{L}_{q}\left(\sigma_{\left.\right|_{A}}\right)_{\left.\right|_{F}}$. Then, $u=v+\sigma_{\left.\right|_{A}}$ where $v \in \mathcal{C}(F)$ is the unique solution of the equation $\mathcal{L}_{q}(v)=f$ on $F$. Since for all $x \in F$,

$$
f(x)=\int_{A} c(x, y) \sigma(y) d y
$$

it follows that $f \in \mathcal{C}^{+}(F)$ and hence $v \in \mathcal{C}^{+}(F)$, which implies that $u \geq 0$. On the other hand, consider $w=\sigma-u$. Then, $w \geq 0$ on $F^{c}, \mathcal{L}_{q}(w)=$ $\left(q-q_{\sigma}\right) \sigma \geq 0$ on $F$ and hence $u \leq \sigma$. On the other hand, we have

$$
\mathcal{L}_{q}(u)(x)=-\int_{V} c(x, y) u(y) d y \leq 0, \quad \text { for all } x \in B,
$$


whereas

$$
\mathcal{L}_{q}(u)(x)=\int_{V} c(x, y)(\sigma(y)-u(y)) d y+\left(q(x)-q_{\sigma}(x)\right) \sigma(x) \geq 0
$$

for all $x \in A$, by equality (2.1).

Finally, if $H$ is a connected component of $F$, then applying the above proposition it follows that either $u=0$ or $u>0$ on $H$. If $u=0$ on $H$, then for all $x \in H$ we get that

$$
\mathcal{L}_{q}(u)(x)=-\int_{A} c(x, y) \sigma(y) d y
$$

which implies that necessarily $\delta(H) \cap A=\emptyset$. That $u<\sigma$ on $H$ when either $\delta(H) \cap B \neq \emptyset$ or $q \neq q_{\sigma}$ on $H$, is deduced in the same way considering now the function $v=\sigma-u$.

Under the hypotheses of the above proposition, $\bar{F}$ is called condenser with positive and negative plates $A$ and $B$, respectively and the boundary value problem is called the condenser problem corresponding to $\bar{F}$. Moreover, we say that the condenser is connected if $F$ is a connected subset. Observe that in the conditions of the condenser principle, when $x \in A$, then $\mathcal{L}_{q}(u)(x)=0$ iff $q(x)=q_{\sigma}(x), d(x, B)>1$ and $u=\sigma$ on $\{y \in F: y \sim x\}$, whereas when $x \in B$, then $\mathcal{L}_{q}(u)(x)=0$ iff $d(A, x)>1$ and $u=0$ on $\{y \in F: y \sim x\}$. Also, it is true that if $H$ is a connected component of $F$, then $\mathcal{L}_{q}(u)>0$ on $\delta(H) \cap A$ when $\delta(H) \cap B \neq \emptyset$ and $\mathcal{L}_{q}(u)<0$ on $\delta(H) \cap B$ when $\delta(H) \cap A \neq \emptyset$.

Corollary 4.12 If $\bar{F}$ is a connected condenser and $u$ is the solution of the corresponding condenser problem, then $0<u<\sigma$ on $F, \mathcal{L}_{q}(u)>0$ on the positive plate and $\mathcal{L}_{q}(u)<0$ on the negative plate.

Next we introduce a concept that is closely related with the condenser problem in the case $q=q_{\sigma}$, namely the effective resistance between two non empty subsets. Fixed $\sigma \in \mathcal{C}_{n}^{*}(V)$, consider $A, B$ two disjoint nonempty subsets of $V$ and $u$ the unique solution of the boundary value problem

$$
\left.\begin{array}{rlrl}
\mathcal{L}_{q_{\sigma}}(u)(x) & =0, & & \text { if } x \in V \backslash\{A, B\}, \\
u(x) & =\sigma(x), & & \text { if } x \in A, \\
u(x) & =0, & & \text { if } x \in B .
\end{array}\right\}
$$

Observe that when $\delta(V \backslash\{A, B\})=A \cup B$, then $V$ is a condenser with plates $A$ and $B$. In this case, the positive plate, $A$, is called source and 
the negative plate, $B$ is called the sink. In any case, the same arguments that in the proof of the condenser principle show that $0 \leq u \leq \sigma$ on $V$, $\mathcal{L}_{q_{\sigma}}(u) \geq 0$ on $A$ and $\mathcal{L}_{q_{\sigma}}(u) \leq 0$ on $B$. Moreover, if $H$ is a connected component of $V \backslash\{A, B\}$, then $u>0$ on $H$ when $\delta(H) \cap A \neq \emptyset, u=0$ on $H$ when $\delta(H) \cap A=\emptyset, u<\sigma$ on $H$ when $\delta(H) \cap B \neq \emptyset$ and $u=\sigma$ on $H$ when $\delta(H) \cap B \neq \emptyset$. In addition, when $x \in A$, then $\mathcal{L}_{q_{\sigma}}(u)(x)=0$ iff $d(x, B)>1$ and $u=\sigma$ on $\{y \in V \backslash\{A, B\}: y \sim x\}$, whereas when $x \in B$, then $\mathcal{L}_{q_{\sigma}}(u)(x)=0$ iff $d(A, x)>1$ and $u=0$ on $\{y \in V \backslash\{A, B\}: y \sim x\}$. It is also true that if $H$ is a connected component of $V \backslash\{A, B\}$, then $\mathcal{L}_{q_{\sigma}}(u)>0$ on $\delta(H) \cap A$ when $\delta(H) \cap B \neq \emptyset$ and $\mathcal{L}_{q_{\sigma}}(u)<0$ on $\delta(H) \cap B$ when $\delta(H) \cap A \neq \emptyset$.

The effective conductance between $A, B$, with respect to $\sigma$, is defined as the value $\mathrm{C}_{\sigma}(A, B)=\mathcal{E}_{q_{\sigma}}(u, u)$. Clearly, $\mathrm{C}_{\sigma}(A, B)>0$, otherwise, $u=a \sigma$ and hence $u$ can not verify $u=0$ on $B$ and $u=\sigma$ on $A$ simultaneously. The effective resistance between $A$ and $B$, w.r.t. $\sigma$, is defined as the value $\mathrm{R}_{\sigma}(A, B)=\mathrm{C}_{\sigma}(A, B)^{-1}$. The effective conductance, and hence the effective resistance, is a symmetric set function, that is, $\mathrm{C}_{\sigma}(A, B)=\mathrm{C}_{\sigma}(B, A)$ since $\mathcal{E}_{q_{\sigma}}(u, u)=\mathcal{E}_{q_{\sigma}}(\sigma-u, \sigma-u)$. So, it is irrelevant which set acts as the source and which set acts as the sink.

On the other hand, applying the Dirichlet principle we obtain that

$$
\mathrm{C}_{\sigma}(A, B)=\min \left\{\mathcal{E}_{q_{\sigma}}(v, v): v=\sigma \text { on } A \text { and } v=0 \text { on } B\right\}
$$

and moreover,

$$
\mathrm{C}_{\sigma}(A, B)=\int_{V} u \mathcal{L}_{q_{\sigma}}(u) d x=\int_{A} \sigma \mathcal{L}_{q_{\sigma}}(u) d x=-\int_{B} \sigma \mathcal{L}_{q_{\sigma}}(u) d x
$$

The special case $q=0$, that is when $\sigma=1$, and both $A$ and $B$ consist of a single vertex has been extensively treated in the literature. Now, defining $R_{\sigma}: V \times V \longrightarrow(0,+\infty)$ as $R_{\sigma}(x, y)=\mathrm{R}_{\sigma}(\{x\},\{y\})$ when $x \neq y$ and $R_{\sigma}(x, x)=0$, it follows that $R_{\sigma}$ is a symmetric function. Moreover, if $u$ is the unique solution of the boundary value problem $\mathcal{L}_{q_{\sigma}}(u)=0$ on $V \backslash\{x, y\}$, $u(x)=\sigma(x)$ and $u(y)=0$, then

$$
1=\sigma(x) R_{\sigma}(x, y) \mathcal{L}_{q_{\sigma}}(u)(x)
$$

and hence

$$
R_{\sigma}(x, y) \mathcal{L}_{q_{\sigma}}(u)=\frac{1}{\sigma}\left(\varepsilon_{x}-\varepsilon_{y}\right) \quad \text { on } V .
$$

We conclude this section with a generalization of a classical result, see for instance [7]. 
Proposition 4.13 Consider $x, y \in V$ and $v \in \mathcal{C}(V)$ any solution of the Poisson equation

$$
\mathcal{L}_{q_{\sigma}}(v)=\frac{1}{\sigma}\left(\varepsilon_{x}-\varepsilon_{y}\right)
$$

Then

$$
R_{\sigma}(x, y)=\mathcal{E}_{q_{\sigma}}(v, v)=\frac{v(x)}{\sigma(x)}-\frac{v(y)}{\sigma(y)}
$$

Proof. Clearly, $v=R_{\sigma}(x, y) u+a \sigma$ with $a \in \mathbb{R}$, where $u$ satisfies $\mathcal{L}_{q_{\sigma}}(u)=0$ on $V \backslash\{x, y\}, u(x)=\sigma(x)$ and $u(y)=0$. This implies that

$$
\mathcal{E}_{q_{\sigma}}(v, v)=R_{\sigma}(x, y)^{2} \mathcal{E}_{q_{\sigma}}(u, u)=R_{\sigma}(x, y) .
$$

Moreover, since $u(y)=0$ and $u(x)=\sigma(x)$, necessarily $a=\frac{v(y)}{\sigma(y)}$ and hence

$$
R_{\sigma}(x, y)=\frac{v(x)}{\sigma(x)}-\frac{v(y)}{\sigma(y)}
$$

\section{Green and Poisson kernels associated with Schrö- dinger operators}

In this section we assume that hypothesis H1 is always satisfied and then, we build the kernels associated with the inverse operators which correspond either to a semihomogeneous Dirichlet problem or to a Poisson equation. In the same way that in the continuous case, such operators will be called Green operators and we will show that they are integral operators. In addition, for any proper subset, we will also consider the kernel associated with the inverse operator of the boundary value problem in which the equation is homogeneous and the boundary data is prescribed. Such integral operator will be called Poisson operator.

Here, we study the properties of the above-mentioned integral operators. Firstly we establish the basic notions about integral operators and their associated kernels. Then, we prove the existence and uniqueness of Green and Poisson operators for each proper subset $F$, we show some of their properties and we build the associated Green or Poisson kernels. On the other hand, under hypothesis H2 we make an analogous study for the Green operator for $V$. Moreover, we extend this work to the singular case, that is, when $F=V$ and $q=q_{\sigma}$ simultaneously and we construct all the Green operators that represent generalized inverses of the problem. In particular, we concentrate on the so-called orthogonal Green operator. Finally, we extend the results on monotonicity of Green kernels with respect to monotone variations of the 0 -order term proved in [20] by M. Yamasaki. 
We start introducing some concepts about kernels and integral operators. We remark that if $F_{1}$ and $F_{2}$ are non empty subsets, $\mathcal{C}\left(F_{1} \times F_{2}\right)$ denotes the set of functions $K \in \mathcal{C}(V \times V)$ such that $K(x, y)=0$ if $(x, y) \notin F_{1} \times F_{2}$.

If $F$ is a non empty subset of $V$, any function $K \in \mathcal{C}(F \times F)$ will be called a kernel on $F$. Of course, if $F \subset H$, then each kernel on $F$ is also a kernel on $H$.

If $K$ is a kernel on $F$, for each $x, y \in F$ we denote by $K^{x}$ and $K_{y}$ the functions of $\mathcal{C}(F)$ defined by $K^{x}(y)=K_{y}(x)=K(x, y)$. Moreover, when $F$ is a proper subset and $K$ is a kernel on $\bar{F}$, for each $x \in \delta(F)$ and each $y \in \bar{F}$, we denote by

$$
\left(\frac{\partial K}{\partial \mathrm{n}}\right)(x, y) \text { the value }\left(\frac{\partial K_{y}}{\partial \mathrm{n}}\right)(x)
$$

whereas for each $x \in \bar{F}$ and each $y \in \delta(F)$ we denote by

$$
\left(\frac{\partial K}{\partial \mathrm{n}_{y}}\right)(x, y) \text { the value }\left(\frac{\partial K^{x}}{\partial \mathrm{n}}\right)(y) .
$$

If $K$ is a kernel on $F$, we define the integral operator associated with $K$ as the endomorphism $\mathcal{K}: \mathcal{C}(F) \longrightarrow \mathcal{C}(F)$ that assigns to each $f \in \mathcal{C}(F)$, the function

$$
\mathcal{K}(f)(x)=\int_{F} K(x, y) f(y) d y \quad \text { for all } x \in V .
$$

The relation between kernels, integral operators and endomorphisms of $\mathcal{C}(F)$ is given by the following result. Its first part can be seen as a discrete version of the Schwartz's Kernel Theorem, because the natural identification between $\mathcal{C}(F)$ and its dual space.

Proposition 5.1 (Kernel Theorem) Each endomorphism of $\mathcal{C}(F)$ is an integral operator associated with a kernel on $F$ which is uniquely determined. Moreover, if $\mathcal{K}$ is an integral operator on $F, K$ is its associated kernel and $A$ is a non empty subset of $F$, then the following statements hold: $\mathcal{K}$ is self-adjoint iff $K$ is symmetric, that is $K(x, y)=K(y, x)$ for all $x, y \in F$, $\operatorname{Img} \mathcal{K} \subset \mathcal{C}(A)$ iff $K \in \mathcal{C}(A \times F)$ and $\mathcal{C}(F \backslash A) \subset \operatorname{ker} \mathcal{K}$ iff $K \in \mathcal{C}(F \times A)$.

Proof. It is clear that if $\mathcal{K}$ is the integral operator associated with the kernel $K$ then $K_{y}=\mathcal{K}\left(\varepsilon_{y}\right)$ for all $y \in F$. Conversely, if $\mathcal{K}$ is an endomorphism of $\mathcal{C}(F)$ and we consider the function $K: F \times F \longrightarrow \mathbb{R}$ given by $K(x, y)=\mathcal{K}\left(\varepsilon_{y}\right)(x)$ for each $x, y \in F$, then $K$ is a kernel on $F$ and $\mathcal{K}$ is the integral operator associated with it. In addition, if $\mathcal{K}$ is a self-adjoint operator, then for all $x, y \in F$ it must be satisfied that

$$
K(x, y)=\mathcal{K}\left(\varepsilon_{y}\right)(x)=\int_{F} \varepsilon_{x} \mathcal{K}\left(\varepsilon_{y}\right) d z=\int_{F} \varepsilon_{y} \mathcal{K}\left(\varepsilon_{x}\right) d z=\mathcal{K}\left(\varepsilon_{x}\right)(y)=K(y, x)
$$

and hence, $K$ is symmetric. 
Conversely, if $K$ is symmetric, then for all $f, g \in \mathcal{C}(F)$ it is verified that

$$
\begin{aligned}
\int_{F} g \mathcal{K}(f) d x & =\int_{F} \int_{F} g(x) K(x, y) f(y) d y d x \\
& =\int_{F} \int_{F} f(y) K(y, x) g(x) d x d y=\int_{F} f \mathcal{K}(g) d y
\end{aligned}
$$

and hence $\mathcal{K}$ is self-adjoint.

On the other hand, let $\mathcal{K}$ be an endomorphism of $\mathcal{C}(F), K$ its associated kernel and $A$ a non empty subset of $F$. If $K \in \mathcal{C}(A \times F)$, then for each $f \in \mathcal{C}(F)$,

$$
\mathcal{K}(f)(x)=\int_{F} K(x, y) f(y) d y=0, \quad \text { for all } x \notin A
$$

and hence $\mathcal{K}(f) \in \mathcal{C}(A)$. Conversely, if Img $\mathcal{K} \subset \mathcal{C}(A)$, then $K(x, y)=$ $\mathcal{K}\left(\varepsilon_{y}\right)(x)=0$ for all $y \in F$ and $x \notin A$ which implies that $K \in \mathcal{C}(A \times F)$.

Finally, if $K \in \mathcal{C}(F \times A)$, then

$$
\mathcal{K}(f)(x)=\int_{A} K(x, y) f(y) d y \quad \text { for all } x \in F
$$

and $f \in \mathcal{C}(F)$ and hence $\mathcal{K}(f)=0$ when $f \in \mathcal{C}(F \backslash A)$. Conversely, if $\mathcal{C}(F \backslash A) \subset \operatorname{ker} \mathcal{K}$, then $K(x, y)=\mathcal{K}\left(\varepsilon_{y}\right)(x)=0$ for all $x \in F$ and $y \notin A$ which implies that $K \in \mathcal{C}(F \times A)$.

Observe that since $\mathcal{C}(A) \subset \mathcal{C}(F)$ when $A \subset F$, the Kernel Theorem implies that each linear operator $\mathcal{K}: \mathcal{C}(F) \longrightarrow \mathcal{C}(A)$ is identified with an integral operator whose kernel $K$ satisfies that $K \in \mathcal{C}(A \times F)$. In addition, if $\mathcal{K}: \mathcal{C}(A) \longrightarrow \mathcal{C}(F)$ is a linear operator, then defining $\mathcal{K}(f)=\mathcal{K}\left(f_{\left.\right|_{A}}\right)$ for all $f \in \mathcal{C}(F)$, the operator has been extended to an endomorphism of $\mathcal{C}(F)$, that we continue calling $\mathcal{K}$, such that $\mathcal{C}(F \backslash A) \subset \operatorname{ker} \mathcal{K}$. Therefore $\mathcal{K}$ is an integral operator whose kernel $K$ satisfies that $K \in \mathcal{C}(F \times A)$.

Now we are ready to introduce the concepts of Green and Poisson operators and kernels. Firstly, we consider the case in which $F$ is a proper subset of $V$. Recall that in this situation, hypothesis $\mathrm{H} 1$ implies that for each $f \in \mathcal{C}(F)$ and each $g \in \mathcal{C}(\delta(F))$ there exists a unique function $u \in \mathcal{C}(\bar{F})$ such that $\mathcal{L}_{q}(u)=f$ on $F$ and $u=g$ on $\delta(F)$. In particular, for each $f \in \mathcal{C}(F)$ there exists a unique function $u \in \mathcal{C}(F)$ such that $\mathcal{L}_{q}(u)=f$ on $F$, whereas for each $g \in \mathcal{C}(\delta(F))$, there exists a unique function $u \in \mathcal{C}(\bar{F})$ such that $\mathcal{L}_{q}(u)=0$ on $F$ and $u=g$ on $\delta(F)$. 
Definition 5.2 Let $F$ be a proper subset of $V$. We call the endomorphism $\mathcal{G}^{F}$ of $\mathcal{C}(F)$ that assigns to each $f \in \mathcal{C}(F)$ the unique function $\mathcal{G}^{F}(f) \in \mathcal{C}(F)$ such that $\mathcal{L}_{q}\left(\mathcal{G}^{F}(f)\right)=f$ on $F$ the Green operator for $F$.

We call the linear operator $\mathcal{P}^{F}: \mathcal{C}(\delta(F)) \longrightarrow \mathcal{C}(\bar{F})$ that assigns to each $g \in \mathcal{C}(\delta(F))$ the unique function $\mathcal{P}^{F}(g) \in \mathcal{C}(\bar{F})$ such that $\mathcal{L}_{q}\left(\mathcal{P}^{F}(g)\right)=0$ on $F$ and $\mathcal{P}^{F}(g)=g$ on $\delta(F)$ the Poisson operator for $F$.

Of course, the Green operator for $F$ is an isomorphism of $\mathcal{C}(F)$ whose inverse is $\mathcal{L}_{q}$. In the following result we investigate formal properties of the Green and Poisson operators.

Proposition 5.3 If $F$ is a proper subset of $V$, then the Green and the Poisson operators for $F$ are formally self-adjoint in the sense that

$$
\begin{aligned}
\int_{F} g \mathcal{G}^{F}(f) d y & =\int_{F} f \mathcal{G}^{F}(g) d y, \quad \text { for all } f, g \in \mathcal{C}(F), \\
\int_{\delta(F)} g \mathcal{P}^{F}(f) d y & =\int_{\delta(F)} f \mathcal{P}^{F}(g) d y, \text { for all } f, g \in \mathcal{C}(\delta(F)) .
\end{aligned}
$$

In addition, if $\mathcal{N}^{F}: \mathcal{C}(\delta(F)) \longrightarrow \mathcal{C}(F)$ is the linear operator given by

$$
\mathcal{N}^{F}(g)=\mathcal{L}(g)_{\left.\right|_{F}}
$$

then $\mathcal{P}^{F}=\mathcal{I}-\mathcal{G}^{F} \circ \mathcal{N}^{F}$, where $\mathcal{I}$ denotes the identity operator on $\mathcal{C}(\delta(F)$ ).

Proof. Given $f, g \in \mathcal{C}(F)$, consider $u=\mathcal{G}^{F}(f)$ and $v=\mathcal{G}^{F}(g)$. Then $u, v \in \mathcal{C}(F)$ and $\mathcal{L}_{q}(u)=f$ and $\mathcal{L}_{q}(v)=g$ on $F$. In addition since $[\mathrm{P}]$ is formally self-adjoint we get that

$$
\int_{F} g \mathcal{G}^{F}(f) d y=\int_{F} u \mathcal{L}_{q}(v) d y=\int_{F} v \mathcal{L}_{q}(u) d y=\int_{F} f \mathcal{G}^{F}(g) d y .
$$

On the other hand, $\mathcal{P}^{F}$ is self-adjoint since it coincides with the identity operator on $\mathcal{C}(\delta(F))$. Finally, if $g \in \mathcal{C}(\delta(F))$ and we consider $u=\mathcal{P}^{F}(g)$, then $u$ is the unique solution of the boundary value problem $\mathcal{L}_{q}(u)=0$ on $F$ and $u=g$ on $\delta(F)$. This problem is equivalent to the semihomogeneous one $\mathcal{L}_{q}(v)=-\mathcal{L}_{q}(g)$ on $F$ with $v \in \mathcal{C}(F)$ and hence $u=$ $g-\mathcal{G}^{F}\left(\mathcal{L}_{q}(g)_{\mid F}\right)$. The result follows taking into account that $\mathcal{L}_{q}(g)_{\left.\right|_{F}}=\mathcal{L}(g)_{\left.\right|_{F}}$ since $\operatorname{supp}(g) \subset \delta(F)$.

By the Kernel Theorem and the subsequent remark, the Green and Poisson operators for $F$ are integral operators on $F$ and $\bar{F}$, respectively, so we can introduce the following concepts. 
Definition 5.4 If $F$ is a proper subset of $V$, we call the Green and Poisson kernel for $F$ the kernels associated with the Green and Poisson operators for $F$, respectively. They will be denoted by $G^{F}$ and $P^{F}$.

It is clear that the last conclusion of the Kernels Theorem implies that $G^{F} \in \mathcal{C}(F \times F)$ and $P^{F} \in \mathcal{C}(\bar{F} \times \delta(F))$. Moreover, if $f \in \mathcal{C}(F)$ and $g \in \mathcal{C}(\delta(F))$, then the functions given by

$$
u(x)=\int_{F} G^{F}(x, y) f(y) d y \quad \text { and } \quad v(x)=\int_{\delta(F)} P^{F}(x, y) g(y) d y, \quad x \in \bar{F}
$$

are the solutions of the semihomogeneous boundary value problems $\mathcal{L}_{q}(u)=$ $f$ on $F, u=0$ on $\delta(F)$ and $\mathcal{L}_{q}(v)=0$ on $F$ and $v=g$ on $\delta(F)$, respectively. In particular, for each $g \in \mathcal{C}(\delta(F))$ we have that

$$
g(x)=\int_{\delta(F)} P^{F}(x, y) g(y) d y \quad \text { for all } x \in \delta(F) .
$$

So, $1=\int_{\delta(F)} P^{F}(x, y) d y$ for all $x \in \delta(F)$ and $\sigma(x)=\int_{\delta(F)} P^{F}(x, y) \sigma(y) d y$ for all $x \in \bar{F}$ when $q=q_{\sigma}$.

Now, the relation between an integral operator and its associated kernel enables us to characterize the Green and Poisson kernels as solutions of suitable boundary value problems.

Proposition 5.5 Let $F$ be a proper subset of $V$ and $G^{F} \in \mathcal{C}(F \times F)$ and $P^{F} \in \mathcal{C}(\bar{F} \times \delta(F))$ the Green and Poisson kernels for $F$. Then for all $y \in F$, the function $G_{y}^{F}$ is characterized by $\mathcal{L}_{q}\left(G_{y}^{F}\right)=\varepsilon_{y}$ on $F$ and for all $y \in \delta(F)$, the function $P_{y}^{F}$ is characterized by $\mathcal{L}_{q}\left(P_{y}^{F}\right)=0$ on $F$ and $P_{y}^{F}=\varepsilon_{y}$ on $\delta(F)$.

Moreover, $G^{F}$ is symmetric on $F$,

$$
P^{F}(x, y)=\varepsilon_{y}(x)-\left(\frac{\partial G^{F}}{\partial \mathrm{n}_{y}}\right)(x, y) \quad \text { for all } x \in \bar{F} \text { and } y \in \delta(F)
$$

and $\frac{\partial P^{F}}{\partial \mathrm{n}}$ is symmetric on $\delta(F)$, that is

$$
\left(\frac{\partial P^{F}}{\partial \mathrm{n}}\right)(x, y)=\left(\frac{\partial P^{F}}{\partial \mathrm{n}}\right)(y, x) \quad \text { for all } x, y \in \delta(F) .
$$

Proof. The symmetry of $G^{F}$ follows directly from the Kernel Theorem. Moreover, for each $y \in F, G_{y}^{F}=\mathcal{G}^{F}\left(\varepsilon_{y}\right)$ and hence $\mathcal{L}_{q}\left(G_{y}^{F}\right)=\varepsilon_{y}$. In the same way if $y \in \delta(F)$, then $P_{y}^{F}=\mathcal{P}^{F}\left(\varepsilon_{y}\right)$ is the unique solution of the boundary value problem $\mathcal{L}_{q}\left(P_{y}^{F}\right)=0$ on $F$ and $P_{y}^{F}=\varepsilon_{y}$ on $\delta(F)$. Fixed $x, y \in \delta(F)$ 
if we consider the functions $u=P_{y}^{F}$ and $v=P_{x}^{F}$, then $\mathcal{L}_{q}(u)=\mathcal{L}_{q}(v)=0$ on $F$ and applying the Second Green Identity

$$
\left(\frac{\partial P^{F}}{\partial \mathrm{n}}\right)(x, y)=\int_{\delta(F)} v \frac{\partial u}{\partial \mathrm{n}} d z=\int_{\delta(F)} u \frac{\partial v}{\partial \mathrm{n}} d z=\left(\frac{\partial P^{F}}{\partial \mathrm{n}}\right)(y, x)
$$

and hence $\frac{\partial P^{F}}{\partial \mathrm{n}}$ is symmetric on $\delta(F)$.

On the other hand, the last part of Proposition 5.3 implies that $P_{y}^{F}=$ $\varepsilon_{y}-\mathcal{G}^{F}\left(\mathcal{L}\left(\varepsilon_{y}\right)_{\left.\right|_{F}}\right)$, for all $y \in \delta(F)$. Since for all $x \in F$,

$$
\mathcal{L}\left(\varepsilon_{y}\right)(x)=\int_{V} c(x, z)\left(\varepsilon_{y}(x)-\varepsilon_{y}(z)\right) d z=-c(x, y),
$$

we get that for all $x \in F$, and all $y \in \delta(F)$,

$$
\begin{aligned}
\mathcal{G}^{F}\left(\mathcal{L}\left(\varepsilon_{y}\right)_{\left.\right|_{F}}\right)(x) & =-\int_{F} G^{F}(x, z) c(z, y) d z \\
& =\int_{F} c(y, z)\left(G^{F}(x, y)-G^{F}(x, z)\right) d z=\left(\frac{\partial G^{F}}{\partial \mathrm{n}_{y}}\right)(x, y)
\end{aligned}
$$

The relation between the Green and Poisson kernels given in the above proposition was obtained in [4], for the case in which $q$ is non negative by using the same technique.

It will be useful to extend the Poisson operator for $F$ to the linear operator $\mathcal{K}^{F}: \mathcal{C}\left(F^{c}\right) \longrightarrow \mathcal{C}(V)$ that assigns to any $g \in \mathcal{C}\left(F^{c}\right)$ the unique solution of the boundary value problem $\mathcal{L}_{q}(u)=0$ on $F$ and $u=g$ on $F^{c}$. Of course, $\mathcal{K}^{F}$ is an integral operator which kernel satisfies $K^{F} \in \mathcal{C}\left(V \times F^{c}\right)$. Now for all $y \in F^{c}, K_{y}^{F}$ is characterized by equations $\mathcal{L}_{q}\left(K_{y}^{F}\right)=0$ on $F$ and $K_{y}^{F}=\varepsilon_{y}$ on $F^{c}$. Therefore, $K_{y}^{F}=P_{y}^{F}$ for all $y \in \delta(F)$ and the solution of the previous boundary value problem is given by

$u(x)=\int_{F^{c}} K^{F}(x, y) g(y) d y=g(x) 1_{\mathrm{Ext}(F)}(x)+\int_{\delta(F)} P^{F}(x, y) g(y) d y$, for all $x \in V$.

In the sequel we will identify $\mathcal{K}^{F}$ and $K^{F}$, with the Poisson operator and the Poisson kernel for $F$, and we will use the notations $\mathcal{P}^{F}$ and $P^{F}$ for them, respectively.

Next, we define the concept of Green operator and Green kernel when $F=V$ both in the case $q \neq q_{\sigma}$ and $q=q_{\sigma}$. For this, we will consider the vectorial subspace $\mathcal{V}=\operatorname{ker}\left(\mathcal{L}_{q}\right)$ and $\pi$ the orthogonal projection on it. Of 
course, $\mathcal{V}$ is trivial and hence $\pi=0$, when $q \neq q_{\sigma}$. Otherwise, $\mathcal{V}$ is the subspace generated by $\sigma$, and hence

$$
\pi(f)=\frac{\sigma}{\|\sigma\|_{2}^{2}} \int_{V} \sigma f d x
$$

Recall that in any case, $\mathcal{L}_{q}$ is an isomorphism of $\mathcal{V}^{\perp}$. Moreover, for each $f \in \mathcal{C}(V)$ there exists $u \in \mathcal{C}(V)$ such that $\mathcal{L}_{q}(u)=f-\pi(f)$ and then $u+\mathcal{V}$ is the set of all functions such that $\mathcal{L}_{q}(v)=f-\pi(f)$.

Definition 5.6 We call any endomorphism $\widetilde{\mathcal{G}}$ of $\mathcal{C}(V)$ that assigns to each $f \in \mathcal{C}(V)$ a function $\widetilde{\mathcal{G}}(f) \in \mathcal{C}(V)$ verifying that $\mathcal{L}_{q}(\widetilde{\mathcal{G}}(f))=f-\pi(f)$ a Green operator for $V$. Moreover, we say that $\widetilde{\mathcal{G}}$ is orthogonal if $\widetilde{\mathcal{G}}(f) \in \mathcal{V}^{\perp}$, for all $f \in \mathcal{C}(V)$.

We call the kernels associated with Green operators for $V$ the Green kernels for $V$. They will be generically denoted by $\widetilde{G}$.

It is clear that in any case there exists a unique orthogonal Green operator for $V$. It will be denoted by $\mathcal{G}$ and it is an isomorphism of $\mathcal{V}^{\perp}$, inverse of $\mathcal{L}_{q}$. In particular, when $q \neq q_{\sigma}$ there exists a unique Green operator for $V$ and therefore it coincides with the orthogonal Green operator for $V$. Since in this case $\mathcal{V}=\{0\}$, the Green operator is an isomorphism of $\mathcal{C}(V)$. The kernel associated with the unique orthogonal Green operator for $V$ will be called the orthogonal Green kernel for $V$ and will be denoted by $G$.

Proposition 5.7 If $\widetilde{\mathcal{G}}$ is any Green operator for $V$, then

$$
\int_{V} g \widetilde{\mathcal{G}}(f) d x=\int_{V} f \widetilde{\mathcal{G}}(g) d x \quad \text { for all } f, g \in \mathcal{V}^{\perp} .
$$

Moreover, the orthogonal Green operator for $V$ is self-adjoint, that is

$$
\int_{V} g \mathcal{G}(f) d x=\int_{V} f \mathcal{G}(g) d x \quad \text { for all } f, g \in \mathcal{C}(V) .
$$

Proof. Let $f, g \in \mathcal{V}^{\perp}$ and consider $u=\widetilde{\mathcal{G}}(f)$ and $v=\widetilde{\mathcal{G}}(g)$. Then $\mathcal{L}_{q}(u)=f, \mathcal{L}_{q}(v)=g$ and since $\mathcal{L}_{q}$ is self-adjoint, then

$$
\int_{V} g \widetilde{\mathcal{G}}(f) d x=\int_{V} u \mathcal{L}_{q}(v) d x=\int_{V} v \mathcal{L}_{q}(u) d x=\int_{V} f \widetilde{\mathcal{G}}(g) d x .
$$

Consider now $f, g \in \mathcal{C}(V), u=\mathcal{G}(f)$ and $v=\mathcal{G}(g)$. Then $u, v \in \mathcal{V}^{\perp}$, $\mathcal{L}_{q}(u)=f-\pi(f), \mathcal{L}_{q}(v)=g-\pi(g)$ and hence

$$
\begin{aligned}
\int_{V} g \mathcal{G}(f) d x & =\int_{V}(g-\pi(g)) u d x=\int_{V} u \mathcal{L}_{q}(v) d x=\int_{V} v \mathcal{L}_{q}(u) d x \\
& =\int_{V}(f-\pi(f)) v d x=\int_{V} f \mathcal{G}(g) d x
\end{aligned}
$$


If $\widetilde{G}$ is a Green kernel for $V$, then $\widetilde{G} \in \mathcal{C}(V \times V)$ and if $f \in \mathcal{C}(V)$ then the function given by

$$
u(x)=\int_{V} \widetilde{G}(x, y) f(y) d y \quad \text { for all } x \in V
$$

is a solution of the Poisson equation $\mathcal{L}_{q}(u)=f-\pi(f)$. In addition, if $\widetilde{G}=G$, then $u$ is the unique solution in $\mathcal{V}^{\perp}$. The relation between an integral operator and its associated kernel enables us again to characterize the Green kernels for $V$ as solutions of suitable boundary value problems.

Proposition 5.8 For all $y \in V$, the function $G_{y}$ is characterized by equations

$$
\mathcal{L}_{q}\left(G_{y}\right)=\varepsilon_{y}-a \sigma(y) \sigma \quad \text { and } \quad a \int_{V} \sigma G_{y} d x=0,
$$

where $a=0$ if $q \neq q_{\sigma}$ and $a=\|\sigma\|_{2}^{-2}$ if $q=q_{\sigma}$. Moreover, $G$ is symmetric and $\widetilde{G}$ is a Green kernel for $V$ iff there exist $\tau \in \mathcal{C}(V)$ such that

$$
\widetilde{G}=G+\sigma \otimes \tau \text {. }
$$

In addition, $\widetilde{G}$ is symmetric iff $\tau$ is a multiple of $\sigma$, that is iff

$$
\widetilde{G}=G+b \sigma \otimes \sigma
$$

where $b \in \mathbb{R}$.

Proof. In any case, for all $y \in V, \widetilde{G}_{y}=\widetilde{\mathcal{G}}\left(\varepsilon_{y}\right)$ and hence $\mathcal{L}_{q}\left(\widetilde{G}_{y}\right)=\varepsilon_{y}-\pi\left(\varepsilon_{y}\right)$. When $q \neq q_{\sigma}$, then $\pi=0$, whereas when $q=q_{\sigma}$, then

$$
\pi\left(\varepsilon_{y}\right)=\frac{\sigma}{\|\sigma\|_{2}^{2}} \int_{V} \sigma \varepsilon_{y} d x=\frac{\sigma(y)}{\|\sigma\|_{2}^{2}} \sigma .
$$

When $\widetilde{G}=G$, it must be also satisfied that $\mathcal{G}\left(\varepsilon_{y}\right) \in \mathcal{V}^{\perp}$, that is

$$
\int_{V} \sigma G_{y} d x=0 \quad \text { for all } y \in V \text {. }
$$

Moreover $G$ is symmetric since $\mathcal{G}$ is self-adjoint.

Suppose that $q=q_{\sigma}$ and consider $\widetilde{G}$ any Green kernel for $V$. Then, for all $f \in \mathcal{C}(V)$

$$
\int_{V}(\widetilde{G}(x, y)-G(x, y)) f(y) d y \in \mathcal{V} .
$$

In particular, taking $f=\varepsilon_{z}$ for each $z \in V$, we get that there exists $\tau(z) \in \mathbb{R}$ such that $\widetilde{G}(x, z)=G(x, z)+\tau(z) \sigma(x)$ for all $x \in V$. Since $G$ is symmetric, it results that $\widetilde{G}$ is symmetric iff $\sigma(x) \tau(y)=\sigma(y) \tau(x)$ for all $x, y \in V$, that is iff $\tau$ is a multiple of $\sigma$. 
Now, we are going to establish new relations between Green and Poisson kernels. With this aim, if $F$ is a nonempty subset, it will be useful to consider for each $y \in \bar{F}$ the set $F_{y}$ defined as follows:

(1) If $F$ is a proper subset and $y \in \delta(F), F_{y}$ is the set of vertices of $F$ that are in the connected component of $F \cup\{y\}$ containing $y$.

(2) If $y \in F, F_{y}$ is the connected component of $F$ containing $y$.

Observe that in both cases, (1) and $(2), \delta\left(F_{y}\right) \subset \delta(F)$ for all $y \in \bar{F}$. Moreover, in case (1), $F_{y}$ is not necessarily a connected set but $y \in \delta(H)$, for any connected component $H$ of $F_{y}$. On the other hand, when $F$ is connected, then $F_{y}=F$ for all $y \in \bar{F}$.

Proposition 5.9 For all $y \in \delta(F)$ it is verified that

$$
\begin{aligned}
& 0<P_{y}^{F} \leq \frac{\sigma}{\sigma(y)} \quad \text { on } F_{y}, \quad P_{y}^{F}=0 \quad \text { on } F \backslash F_{y}, \\
& \mathcal{L}_{q}\left(P_{y}^{F}\right)<0 \quad \text { on } \delta\left(F_{y}\right) \backslash\{y\} \quad \text { and } \quad \mathcal{L}_{q}\left(P_{y}^{F}\right)(y)>0,
\end{aligned}
$$

except when $F=V \backslash\{y\}$ and $q=q_{\sigma}$ simultaneously, in which case $P_{y}^{F}=\frac{\sigma}{\sigma(y)}$ and hence $\mathcal{L}_{q}\left(P_{y}^{F}\right)(y)=0$. In addition, if $H$ is a connected component of $F_{y}$ and either $|\delta(H)|>1$ or $q \neq q_{\sigma}$ on $H$, then $P_{y}^{F}<\frac{\sigma}{\sigma(y)}$ on $H$, whereas if $\delta(H)=\{y\}$ and $q=q_{\sigma}$ on $H$ simultaneously, then $P_{y}^{F}=\frac{\sigma}{\sigma(y)}$ on $H$.

Proof. Let $y \in \delta(F)$ and consider the sets $A=\{y\}$ and $B=\delta(F) \backslash\{y\}$ and the function $u=\sigma(y) P_{y}^{F}$. Then $u$ is the unique solution of the condenser problem for $\bar{F}$ with plates $A$ and $B$. Therefore, $0<u \leq \sigma$ on $F_{y}, u=0$ on $F \backslash F_{y}, \mathcal{L}_{q}(u)(y) \geq 0, \mathcal{L}_{q}(u) \leq 0$ on $\delta(F) \backslash\{y\}$ and $\mathcal{L}_{q}(u)<0$ on $\delta\left(F_{y}\right) \backslash\{y\}$ since $\delta(H) \cap A \neq \emptyset$ for any connected component of $F_{y}$. Moreover,

$$
\delta(H) \cap B \neq \emptyset \quad \text { iff } \quad|\delta(H)|>1,
$$

so the last conclusion follows from the condenser principle.

Finally, since

$$
\mathcal{L}_{q}(u)(y) \sigma(y)=\int_{V} \mathcal{L}_{q}(u) u d x=\mathcal{E}_{q}(u, u)
$$

we get that $\mathcal{L}_{q}(u)(y)=0$ iff either $u=0$ when $q \neq q_{\sigma}$ or $u=\alpha \sigma$ when $q=q_{\sigma}$. Since $u(y)=\sigma(y)>0$ and $u=0$ on $F^{c} \backslash\{y\}$, it results that $\mathcal{L}_{q}(u)(y)>0$ except when $q=q_{\sigma}$ and $F=V \backslash\{y\}$ simultaneously.

For the sake of completeness, the Green kernel for $V$ will be denoted by $G^{V}$ in the following proposition. 
Proposition 5.10 Let $F$ be a non empty subset of $V$ and suppose that hypotheses $\mathrm{H} 1$ and $\mathrm{H} 2$ are in force. Then, for all $y \in F$ it is verified that

$$
G_{y}^{F}(y)>0, \quad P_{y}^{F \backslash\{y\}}=\frac{G_{y}^{F}}{G_{y}^{F}(y)} \quad \text { and } \quad 0 \leq G_{y}^{F} \leq G_{y}^{F}(y) \frac{\sigma}{\sigma(y)} \quad \text { on } F .
$$

Moreover, $G_{y}^{F}=\frac{\sigma}{a \sigma(y)}$ iff $F=V$ and $q=a \varepsilon_{y}+q_{\sigma}$ with $a>0$, simultaneously. Otherwise, $G_{y}^{F}=0$ on $F \backslash F_{y}$ and

$$
0<G_{y}^{F}<G_{y}^{F}(y) \frac{\sigma}{\sigma(y)} \quad \text { on } F_{y} \backslash\{y\} .
$$

Proof. From the characterization of $G_{y}^{F}$ and Corollary 4.3, we obtain that $0 \leq G_{y}^{F}$ on $F$ and moreover, $G_{y}^{F}(y)>0$, for all $y \in F$.

For fixed $y \in F$, the function $u=\frac{1}{G_{y}^{F}(y)} G_{y}^{F}$ satisfies that $u(y)=1, u=0$ on $\delta(F)$ and $\mathcal{L}_{q}(u)=\varepsilon_{y}$ on $F$, which implies that $\mathcal{L}_{q}(u)=0$ on $F \backslash\{y\}$, $u=0$ on $\delta(F \backslash\{y\}) \backslash\{y\}$ and $u(y)=1$ and therefore $u=P_{y}^{F \backslash\{y\}}$.

Finally, keeping in mind that if $H=F \backslash\{y\}$, then $\delta\left(H_{y}\right)=\{y\}$ iff $F=V$, the rest of claims are deduced directly from the properties of the Poisson kernel given in the above proposition.

Corollary 5.11 Consider $F$ a non empty and connected subset and suppose that hypotheses $\mathrm{H} 1$ and $\mathrm{H} 2$ are in force. Then, for all $y \in F, G_{y}^{F}$ is strictly positive, $\frac{1}{\sigma} G_{y}^{F}$ takes its maximum value at $\{y\}$ and such a maximum is strict except when $F=V$ and $q=q_{\sigma}$ on $V \backslash\{y\}$ simultaneously, in which case $\frac{1}{\sigma} G^{F}$ is constant.

We finish this section by proving the announced monotonicity properties of Green kernels w.r.t. to the 0-order term, which generalize those obtained in [20]. Of course, these properties can be easily reformulated in terms of Poisson kernels since both types of kernels are related by the expressions given in Proposition 5.10.

Proposition 5.12 Let $F$ be a non empty subset of $V$ and suppose that hypotheses $\mathrm{H} 1$ and $\mathrm{H} 2$ hold. Then

$$
\int_{V} G_{y}^{F} q d x \leq 1 \quad \text { for all } y \in F,
$$

with strict inequality when $F \neq V$. In addition if $\left\{q_{k}\right\}_{k=1}^{\infty}$ satisfy that $q_{k} \downarrow q$ (respectively $q_{k} \uparrow q$ with $q_{1} \geq q_{\sigma}$ and $q_{1} \neq q_{\sigma}$ when $F=V$ ) and for all $k \in \mathbb{N}^{*}, G_{k}^{F}$ denotes the Green kernel for $F$ associated with the operator $\mathcal{L}_{q_{k}}$, then $G_{k}^{F} \uparrow G^{F}$ (respectively $G_{k}^{F} \downarrow G^{F}$ ). 
Proof. Fixed $y \in F$, if $v=G_{y}^{F}$, then $v \in \mathcal{C}^{+}(F)$. Therefore, if $F$ is a proper subset then $\mathcal{L}_{q}(v) \leq 0$ on $F^{c}$ and necessarily $\mathcal{L}_{q}(v)(x)<0$ for some $x \in \delta(F)$. On the other hand,

$\int_{F} G_{y}^{F} q d x=\int_{F}\left(\varepsilon_{y}-\mathcal{L}(v)\right) d x=1-\int_{V} \mathcal{L}(v) d x+\int_{\delta(F)} \mathcal{L}(v) d x=1+\int_{\delta(F)} \mathcal{L}(v) d x$

and the first claim follows observing that the value of $\int_{\delta(F)} \mathcal{L}_{q}(v) d x$ is zero when $F=V$ and strictly negative otherwise.

Now, for each $k \in \mathbb{N}^{*}$ consider $v_{k}=G_{k y}^{F}$ and take $u=G_{x}^{F}$ for fixed $x \in F$. Then applying the Second Green Identity, we obtain that

$$
\begin{aligned}
v_{k}(x) & =\int_{F} \mathcal{L}_{q}(u) v_{k} d z=\int_{F} u \mathcal{L}_{q}\left(v_{k}\right) d z=\int_{F} u \mathcal{L}\left(v_{k}\right) d z+\int_{F} q u v_{k} d z, \\
u(y) & =\int_{F} \mathcal{L}_{q_{k}}\left(v_{k}\right) u d z=\int_{F} \mathcal{L}\left(v_{k}\right) u d z+\int_{F} q_{k} v_{k} u d z .
\end{aligned}
$$

Taking into account that

$$
u(y)=G^{F}(y, x)=G^{F}(x, y)=v(x),
$$

from the above identities we obtain that

$$
v(x)-v_{k}(x)=\int_{V}\left(q_{k}-q\right) v_{k} u d z .
$$

When $q_{k} \downarrow q$, Corollary 4.2 assures that $v \geq v_{k+1} \geq v_{k}$. Moreover, if $\alpha \geq 0$ is such that $G^{F} \leq \alpha$, then $u, v \leq \alpha$ and hence

$$
0 \leq v(x)-v_{k}(x) \leq \alpha^{2} \int_{V}\left(q_{k}-q\right) d z
$$

which implies that $v_{k} \uparrow v$.

When $q_{k} \uparrow q$, then hypothesis $q_{1} \geq q_{\sigma}$ with $q_{1} \neq q_{\sigma}$ if $F=V$, ensures that $G_{k}^{F}$ makes sense for all $k$. Moreover applying again Corollary 4.2 we obtain that $v \leq v_{k} \leq v_{k-1}$. Therefore, if $\alpha \geq 0$ is such that $G_{1}^{F} \leq \alpha$, then

$$
0 \leq v_{k}(x)-v(x) \leq \alpha^{2} \int_{V}\left(q-q_{k}\right) d z,
$$

which implies $v_{k} \downarrow v$.

The first claim in the above proposition is also true for any Green kernel when $F=V$ and $q=q_{\sigma}$ simultaneously, since in this case

$$
\int_{V} \widetilde{G}_{y} q_{\sigma} d x=\int_{V}\left(\varepsilon_{y}-\frac{\sigma(y)}{\|\sigma\|_{2}^{2}} \sigma\right) d x-\int_{V} \mathcal{L}\left(\widetilde{G}_{y}\right) d x=1-\frac{\sigma(y)}{\|\sigma\|_{2}^{2}} \int_{V} \sigma d x<1 .
$$




\section{The kernel associated to a Schrödinger operator}

As we have shown the discrete Schrödinger operators are difference operators verifying analogous properties to those satisfied by self-adjoint second order elliptic operators. In particular, they have associated quadratic forms and resolvents or Green kernels. The aim of this section is to treat the discrete Schrödinger operators from another point of view, which does not seem to have a continuous counterpart. Since any Schrödinger operator, $\mathcal{L}_{q}$, is an endomorphism of $\mathcal{C}(V)$, from the Kernel Theorem it can be seen as an integral operator whose associated kernel is given by $L_{q}(x, y)=\mathcal{L}_{q}\left(\varepsilon_{y}\right)(x)$ for all $x, y \in V$. Therefore, $L_{q}(x, y)=-c(x, y)$ if $x \neq y$ and $L_{q}(x, x)=$ $k(x)+q(x)$ and hence

$$
\mathcal{L}_{q}(u)(x)=\int_{V} L_{q}(x, y) u(y) d y \quad \text { for all } \quad u \in \mathcal{C}(V) .
$$

By considering the kernel $L_{q}$ we build a Potential Theory with respect to it. Of course the kernel $L_{q}$ must satisfy some properties that justify this new point of view. For that, we assume again that hypothesis H1 holds. In particular, the principles satisfied by $L_{q}$ will allow to build the so-called equilibrium measures. The fundamental result of this section consists of obtaining systematically explicit expressions for the Green and Poisson kernels by means of equilibrium measures. Therefore, the obtained results appear as a generalization of those given by authors in $[4,5]$ when $q \in \mathcal{C}^{+}(V)$.

To develop a Potential Theory w.r.t. the kernel $L_{q}$, we next introduce some concepts and notations within this framework. Here we will make an extensive use of the natural identification between functions and Radon measures on $V$, since the underlying space, $V$, is finite. So, for each $u \in \mathcal{C}(V)$ we call mass of $u$ the value

$$
\|u\|=\int_{V} u d x
$$

and for each non empty subset $F$, we denote by

$$
\mathcal{M}^{1}(F)=\left\{u \in \mathcal{C}^{+}(F):\|u\|=1\right\} .
$$

In addition, we call potential and energy of $u$ (w.r.t. $L_{q}$ ) the function and the value given respectively by

$$
\mathcal{L}_{q}(u)(x)=\int_{V} L_{q}(x, y) u(y) d y \text { and } \int_{V} \mathcal{L}_{q}(u) u d x .
$$

Clearly, for each $u \in \mathcal{C}(V)$, the energy of $u$ coincides with the value $\mathcal{E}_{q}(u, u)$. Now we investigate on the properties satisfied by the potentials and by the energy w.r.t. the kernel $L_{q}$. 
Proposition 6.1 (Energy principle) The energy is an strictly convex functional on $\mathcal{M}^{1}(V)$.

Proof. Observe that the verification of the energy principle is equivalent to the property $\mathcal{E}_{q}(u-v, u-v) \geq 0$ for each $u, v \in \mathcal{M}^{1}(V)$, with equality iff $u=v$, that is, it is equivalent to the positive definiteness of $\mathcal{E}_{q}$ on the subspace of functions with null mass.

From Corollary 3.4, $\mathcal{E}_{q}$ is positive semidefinite on $\mathcal{C}(V)$ and positive definite when $q \neq q_{\sigma}$. Moreover, when $q=q_{\sigma}, \mathcal{E}_{q}(w, w)=0$ iff $w=a \sigma$ with $a \in \mathbb{R}$ and hence $a=0$ when $\|w\|=0$.

By applying Corollary 4.3 to the case $f=1_{F}$, we get the following result.

Proposition 6.2 (Equilibrium principle) Let $F$ be a non empty subset of $V$ and suppose that hypothesis $\mathrm{H} 2$ is also verified. Then, there exists a unique $\nu^{F} \in \mathcal{C}^{+}(F)$ such that $\mathcal{L}_{q}\left(\nu^{F}\right)=1_{F}$ on $F$. In addition, $\operatorname{supp}\left(\nu^{F}\right)=F$.

Under the hypotheses of the above proposition, $\nu^{F}$ will be called the equilibrium measure for $F$ (w.r.t. $L_{q}$ ) and it is clear that $\mathcal{E}_{q}\left(\nu^{F}, \nu^{F}\right)=\left\|\nu^{F}\right\|$. Observe that each proper subset of $V$ has an equilibrium measure w.r.t. $L_{q}$, whereas $V$ has an equilibrium measure only when $q \neq q_{\sigma}$. Moreover, it is easy to verify that

$$
\mathcal{L}_{q}\left(\nu^{F}\right)=1_{F}-\left(\int_{F} c(\cdot, y) \nu^{F}(y) d y\right) 1_{\delta(F)}
$$

for any proper subset $F$.

Next, we calculate the equilibrium measure for some simple cases. If $F=\{x\}$, then its equilibrium measure must be a positive multiple of $\varepsilon_{x}$. Moreover, since $\mathcal{L}_{q}\left(\varepsilon_{x}\right)(x)=k(x)+q(x)$ we get that

$$
\nu^{\{x\}}=\frac{1}{k+q} \varepsilon_{x} .
$$

On the other hand, if $q \neq q_{\sigma}$, then the equilibrium measure for $V$ is a positive multiple of $\sigma$, say $\nu^{V}=a \sigma$, iff $q=q_{\sigma}+\frac{1}{a \sigma}$, because $\mathcal{L}_{q}(\sigma)=\left(q-q_{\sigma}\right) \sigma$. In particular, the equilibrium measure for $V$ is constant iff $q$ is a positive constant and in this case, $\nu^{V}=\frac{1}{q}$. Other not so elementary examples as the equilibrium measures for proper subsets of cycles, paths and complete graphs when $q=0$, can be found in [3].

We pay special attention at the equilibrium measures for sets with cardinality $n-1$ because they are important in applications. So, for each $x \in V$ we will denote by $\nu_{x}$ the equilibrium measure for $F=V \backslash\{x\}$. Sometimes, we can determine the explicit value of $\nu_{x}$, for instance this is the case of 
distance-regular graphs which has been used at the beginning of this paper. In any case, we have that for all $x \in V$,

$$
\|\sigma\|+\left(\mathcal{L}_{q}\left(\nu_{x}\right)(x)-1\right) \sigma(x)=\int_{V} \mathcal{L}_{q}\left(\nu_{x}\right) \sigma d y=\int_{V} \mathcal{L}_{q}(\sigma) \nu_{x} d y=\int_{V} \nu_{x} \sigma\left(q-q_{\sigma}\right) d y
$$

and therefore,

$$
\mathcal{L}_{q}\left(\nu_{x}\right)=1-\frac{1}{\sigma}\left(\int_{V} \nu_{x} \sigma\left(q-q_{\sigma}\right) d y+\|\sigma\|\right) \varepsilon_{x} .
$$

In particular, when $q=q_{\sigma}$, we have that

$$
\mathcal{L}_{q_{\sigma}}\left(\nu_{x}\right)=1-\frac{\|\sigma\|}{\sigma} \varepsilon_{x} .
$$

The last property of kernel $L_{q}$ we analyze here is related to the maximum value of its potentials. We emphasize that this property must not be confused with the maximum principle for $\mathcal{L}_{q}$ as an operator (Corollary 4.5).

Proposition 6.3 (Frostman's maximum principle) For all non-zero $u \in$ $\mathcal{C}^{+}(V)$, we have

$$
\max _{x \in V}\left\{\mathcal{L}_{q}(u)(x)\right\}=\max _{x \in \operatorname{supp}(u)}\left\{\mathcal{L}_{q}(u)(x)\right\}
$$

Proof. Consider a non-zero $u \in \mathcal{C}^{+}(V)$. If $x \notin \operatorname{supp}(u)$ we get that $\mathcal{L}_{q}(u)(x) \leq 0$, since $u$ is non negative. On the other hand, if $x^{*} \in \operatorname{supp}(u)$ is such that

$$
\frac{u\left(x^{*}\right)}{\sigma\left(x^{*}\right)}=\max _{y \in \operatorname{supp}(u)}\left\{\frac{u(y)}{\sigma(y)}\right\},
$$

then $\mathcal{L}_{q}(u)\left(x^{*}\right) \geq 0$ from expression (2.1) and therefore, the result follows.

In the framework of Potential Theory, the equilibrium principle is often obtained as a consequence of the verification of the energy and the Frostman's maximum principles (see for instance [3].) Moreover, both principles can be used to obtain the equilibrium measures from the solution of either a quadratic and convex programming problem or of a linear programming problem. Specifically, we have that

$$
\min _{u \in \mathcal{M}^{1}(F)}\left\{\mathcal{E}_{q}(u, u)\right\}=\min _{u \in \mathcal{M}^{1}(F)} \max _{x \in F}\left\{\mathcal{L}_{q}(u)(x)\right\}=\min _{\substack{\left.u \in \mathcal{M}^{1}(F) \\ \mathcal{L}_{q}(u)\right|_{F} \leq a 1_{F}}}\{a\} .
$$

If $u^{F}$ is the unique solution of the above problems, then the value $\operatorname{cap}_{q}(F)=$ $\mathcal{E}_{q}\left(u^{F}, u^{F}\right)^{-1}$ is called the Wiener capacity of $F$ (w.r.t. $\left.L_{q}\right)$. In addition, 
the equilibrium measure for $F$ satisfies $\nu^{F}=\operatorname{cap}_{q}(F) u^{F}$, which implies that $\left\|\nu^{F}\right\|=\operatorname{cap}_{q}(F)$. Therefore, if $F$ is a proper subset of $V$ then $0<$ $\operatorname{cap}_{q}(F)<+\infty$. On the other hand, if $q=q_{\sigma}$, then $u^{V}=\|\sigma\|^{-1} \sigma$ and hence $\operatorname{cap}_{q}(V)=+\infty$, whereas if $q \neq q_{\sigma}$, then $\operatorname{cap}_{q}(V)<+\infty$. In particular, when $q=q_{\sigma}+\frac{1}{a \sigma}$ we get that $\operatorname{cap}_{q}(V)=a\|\sigma\|$ and $\operatorname{cap}_{q}(V)=\frac{n}{q}$ when $q$ is a positive constant.

We must note that the Wiener capacity w.r.t. kernel $L_{q}$ is not the capacity usually associated with Schrödinger operators, that is the capacity w.r.t, the associated Dirichlet form. There exist great differences between both types of capacities. For instance, the capacity associated with Dirichlet forms is subadditive and in fact strongly subadditive, whereas the Wiener capacity is not subadditive since it is associated with a signed kernel. In contrast, the Wiener capacity w.r.t. $L_{q}$ retains geometric information on the subsets of $V$, in the sense that it takes into account the adjacency between subsets as the following results show.

Proposition 6.4 Let $F \subset V$ and suppose that hypothesis $\mathrm{H} 2$ is also verified. If $H$ is a proper subset of $F$, then $\nu^{H} \leq \nu^{F}$. Moreover, if $x \in H$ then $\nu^{H}=\nu^{F}$ on $F_{x}$ when $F_{x} \subset H$ and $\nu^{H}<\nu^{F}$ on $F_{x}$, otherwise.

Proof. If $u=\nu^{F}-\nu^{H}$, then $u \in \mathcal{C}(\bar{F}), \mathcal{L}_{q}(u)=1-\mathcal{L}_{q}\left(\nu^{H}\right) \geq 0$ on $F$ and $u=0$ on $\delta(F)$. Therefore, by applying Proposition 4.10 we get that $u \geq 0$ on $F$ and for all $x \in H$ either $u=0$ on $F_{x}$ or $u>0$ on $F_{x}$. When $F_{x} \cap(F \backslash H) \neq \emptyset$, the result follows since $u>0$ on $F \backslash H$. When $F_{x} \subset H$, then for each $z \in F_{x}$ we get that $c(z, y)=0$ for all $y \in F \backslash F_{x}$. Therefore, for all $z \in F_{x}$

$$
\begin{aligned}
\mathcal{L}_{q}\left(u_{\left.\right|_{F_{x}}}\right)(z)= & 1 \mathcal{L}_{q}\left(\nu^{F}\right)(z)-\mathcal{L}_{q}\left(\nu^{H}\right)(z) \\
& +\int_{F \backslash F_{x}} c(z, y) \nu^{F}(y) d y-\int_{H \backslash F_{x}} c(z, y) \nu^{H}(y) d y=0,
\end{aligned}
$$

which implies that $u=0$ on $F_{x}$.

Corollary 6.5 Let $F \subset V$ and suppose that hypothesis $\mathrm{H} 2$ is also verified. If $F_{1}, F_{2}$ is a partition of $F$, then $\nu^{F_{1}}+\nu^{F_{2}} \leq \nu^{F}$ and the equality holds iff $d\left(F_{1}, F_{2}\right)>1$.

Corollary 6.6 The Wiener capacity is an strictly increasing set function. Moreover, if $F_{1}, F_{2}$ is a partition of $F$, then $\operatorname{cap}_{q}\left(F_{1}\right)+\operatorname{cap}_{q}\left(F_{2}\right) \leq \operatorname{cap}_{q}(F)$ and the equality holds iff $d\left(F_{1}, F_{2}\right)>1$. In particular, for each subset $F$,

$$
\sum_{x \in F} \frac{1}{k(x)+q(x)} \leq \operatorname{cap}_{q}(F)
$$

with equality iff no two vertices in the subset $F$ represent an edge. 
Now, we are going to obtain the expression of the Green and Poisson kernels associated with the Schrödinger operator $\mathcal{L}_{q}$ by means of equilibrium measures. For the moment and until otherwise be specified, we suppose that hypotheses $\mathrm{H} 1$ and $\mathrm{H} 2$ are true and again we denote by $G^{V}$ the Green kernel for $V$.

Proposition 6.7 Let $F$ be a non empty subset of $V$. Then the following properties hold:

i) The Green kernel for $F$ is given by the expression

$$
G^{F}(x, y)=\left\|\nu^{F}-\nu^{F \backslash\{y\}}\right\|^{-1} \nu^{F}(y)\left(\nu^{F}(x)-\nu^{F \backslash\{y\}}(x)\right) \text {, for all } x, y \in F .
$$

ii) If $F$ is a proper subset and it is not simultaneously true that $|F|=n-1$ and $q=q_{\sigma}$, then the Poisson kernel for $F$ is given by the expression

$$
P^{F}(x, y)=\left(\nu^{F \cup\{y\}}(y)\right)^{-1}\left(\nu^{F \cup\{y\}}(x)-\nu^{F}(x)\right), \text { for all } x, y \in V .
$$

Proof. (i) We know that for all $y \in F, G_{y}^{F}$ is characterized by $\mathcal{L}_{q}\left(G_{y}^{F}\right)=\varepsilon_{y}$ on $F$ and $G_{y}^{F}=0$ on $\delta(F)$.

Consider now $K \in \mathcal{C}(V \times V)$ the function given by $K(x, y)=\nu^{F}(x)-$ $\nu^{F \backslash\{y\}}(x)$ for all $x, y \in V$. Since $K_{y}=K^{x}=0$ on $F^{c}$ because $\operatorname{supp}\left(\nu^{F}\right)$, $\operatorname{supp}\left(\nu^{F \backslash\{y\}}\right) \subset F$ and $\nu^{F}=\nu^{F \backslash\{y\}}$ when $y \notin F$, it follows that $K$ is a kernel on $F$. Moreover

$$
\mathcal{L}_{q}\left(K_{y}\right)(x)=\mathcal{L}_{q}\left(\nu^{F}\right)(x)-\mathcal{L}_{q}\left(\nu^{F \backslash\{y\}}\right)(x)=\left\{\begin{array}{cl}
0, & \text { if } x \neq y, \\
1-\mathcal{L}_{q}\left(\nu^{F \backslash\{y\}}\right)(y), & \text { if } x=y
\end{array}\right.
$$

and the value of $1-\mathcal{L}_{q}\left(\nu^{F-\{y\}}\right)(y)$ can be obtained by applying the Second Green Identity to $\nu^{F \backslash\{y\}}$ and $\nu^{F}$ in the following manner

$$
\begin{aligned}
\left\|\nu^{F \backslash\{y\}}\right\| & =\int_{V} \nu^{F \backslash\{y\}} \mathcal{L}_{q}\left(\nu^{F}\right) d z=\int_{V} \mathcal{L}_{q}\left(\nu^{F \backslash\{y\}}\right) \nu^{F} d z \\
& =\left\|\nu^{F}\right\|-\nu^{F}(y)\left(1-\mathcal{L}_{q}\left(\nu^{F \backslash\{y\}}\right)(y)\right) .
\end{aligned}
$$

(ii) Since for all $y \in F, G_{y}^{F}(y)=\left\|\nu^{F}-\nu^{F \backslash\{y\}}\right\|^{-1} \nu^{F}(y)^{2}$, we obtain that

$$
\frac{G_{y}^{F}}{G_{y}^{F}(y)}=\left(\nu^{F}(y)\right)^{-1}\left(\nu^{F}-\nu^{F \backslash\{y\}}\right) .
$$

So, for $(x, y) \in \bar{F} \times \delta(F)$, the result follows from the relation between $P_{y}^{F}$ and $G_{y}^{F \cup\{y\}}$ given in Corollary 5.10. 
We conclude the proof observing that when $y \in F, F \cup\{y\}=F$, which implies that $\nu^{F \cup\{y\}}=\nu^{F}$, whereas when $y \in \operatorname{Ext}(F), \nu^{F \cup\{y\}}=\nu^{F}+\nu^{\{y\}}$. Hence the value of the given expression for $P_{y}^{F}$ is 0 when $y \in F$ and it is

$$
\frac{\nu^{\{y\}}(x)}{\nu^{\{y\}}(y)}=\varepsilon_{y}(x)
$$

when $y \in \operatorname{Ext}(F)$.

Observe that the expression for $G^{F}$ given in part (i) of the above proposition implies that $G_{y}^{F} \leq \nu^{F}(y)$ for all $y \in F$. In addition, this formula for the Green kernel remains true for $x \in V$, since $\nu^{F}(x)-\nu^{F \backslash\{y\}}(x)=0$ when $x \in F^{c}$, but it can not be extended to $y \in F^{c}$, since $\left\|\nu^{F}-\nu^{F \backslash\{y\}}\right\|=0$. However, we can give an alternative expression for $G^{F}$ which is valid for $x, y \in V$. Specifically, from the proof of above proposition and taking into account that

$$
\mathcal{L}_{q}\left(\nu^{F \backslash\{y\}}\right)(y)=-\int_{V} c(y, z) \nu^{F \backslash\{y\}}(z) d z \leq 0, \quad \text { for all } y \in V,
$$

we obtain

$$
G^{F}(x, y)=\left(1+\int_{V} c(y, z) \nu^{F \backslash\{y\}}(z) d z\right)^{-1}\left(\nu^{F}(x)-\nu^{F \backslash\{y\}}(x)\right),
$$

for all $x, y \in V$.

We have just proved that for any nonempty subset $F$, its Green kernel can be expressed by means of equilibrium measures. Conversely, the equilibrium measure for a subset can be obtained by means of its Green kernel. Specifically, we have that

$$
\nu^{F}(x)=\int_{F} G^{F}(x, y) d y, \quad \text { for all } x \in V
$$

and hence

$$
\operatorname{cap}_{q}(F)=\int_{F \times F} G^{F}(x, y) d x d y .
$$

This fact, together with the symmetry of the Green kernel, allows us to deduce monotonicity properties of the equilibrium measures w.r.t. the 0order term from those verified by Green kernels given in Proposition 5.12.

Corollary 6.8 Consider $F$ a nonempty subset of $V$ and a sequence $\left\{q_{k}\right\}_{k=1}^{\infty}$ in $\mathcal{C}(V)$ such that $q_{k} \downarrow q$, (respectively, $q_{k} \uparrow q$ with $q_{1} \geq q_{\sigma}$ and $q_{1} \neq q_{\sigma}$ when $F=V)$. If for each $k \in \mathbb{N}^{*}$ we denote by $\nu_{k}^{F}$ the equilibrium measure for $F$ w.r.t. the kernel $L_{q_{k}}$, then $\nu_{k}^{F} \uparrow \nu^{F}$ and $\operatorname{cap}_{q_{k}}(F) \uparrow \operatorname{cap}_{q}(F)$ (respectively, $\nu_{k}^{F} \downarrow \nu^{F}$ and $\left.\operatorname{cap}_{q_{k}}(F) \downarrow \operatorname{cap}_{q}(F)\right)$. 
In addition, from the properties of the Green kernels contained in Proposition 5.10, we can deduce some relations between the equilibrium measures $\nu^{F}$ and $\nu^{F \backslash\{y\}}$ when $y \in F$.

Corollary 6.9 Let $F$ be a non empty set. Then, for all $y \in F$ we get that

$$
\nu^{F \backslash\{y\}} \leq \nu^{F} \leq \nu^{F \backslash\{y\}}+\frac{\nu^{F}(y)}{\sigma(y)} \sigma .
$$

Moreover, $\nu^{F-\{y\}}=\nu^{F}$ on $F \backslash F_{y}$ and

$$
\nu^{F \backslash\{y\}}<\nu^{F}<\nu^{F \backslash\{y\}}+\frac{\nu^{F}(y)}{\sigma(y)} \sigma \quad \text { on } F_{y} \backslash\{y\},
$$

except when $F=V$ and $q-q_{\sigma}=a \varepsilon_{y}, a>0$ in which case

$$
\nu^{V}=\nu_{y}+\frac{\nu^{V}(y)}{\sigma(y)} \sigma
$$

In particular, if $F$ is connected, then

$$
\nu^{F \backslash\{y\}}<\nu^{F}<\nu^{F \backslash\{y\}}+\frac{\nu^{F}(y)}{\sigma(y)} \sigma \quad \text { on } F \backslash\{y\},
$$

except when $F=V$ and $q-q_{\sigma}=a \varepsilon_{y}, a>0$.

Of course, we can obtain an analogous relation between $\nu^{F}$ and $\nu^{F \cup\{y\}}$ for $y \in \delta(F)$, considering the expression for the Poisson kernel obtained in the above proposition. The above corollary reaffirms that the equilibrium measure contains global information on the connectivity between vertices of $F$. So, if we connect or disconnect a single vertex to a fixed connected set, then the equilibrium measure of the new subset takes at each vertex a different value.

The expression of the Green kernel for $F$ that appears in the Proposition 6.7 is analogous to that obtained in [4] for the case in which $\sigma$ is a constant function, that is, when $q \in \mathcal{C}^{+}(V)$. Moreover, in the mentioned work the equilibrium measures of subsets with cardinality $n-1$, that is the measures $\nu_{x}, x \in V$, allowed us to obtain a formula for the orthogonal Green kernel for $V$ in the case $q=0$. Now, we are concerned with the general singular case, that is when $F=V$ and $q=q_{\sigma}$ simultaneously for arbitrary $\sigma \in \mathcal{C}^{*}(V)$. Our aim is to express all Green kernels for $V$, and in particular the orthogonal Green kernel for $V$ by means of equilibrium measures and the eigenfunction $\sigma$. 
Proposition 6.10 Suppose that $q=q_{\sigma}$ and consider for each $y \in V$, $u_{y}$ the unique solution of the Dirichlet problem $\mathcal{L}_{q_{\sigma}}(u)=\sigma$ on $V \backslash\{y\}$. Then, any Green kernel for $V$ is given by the formula

$$
\widetilde{G}(x, y)=-\frac{1}{\|\sigma\|_{2}^{2}} u_{y}(x) \sigma(y)+\sigma(x) \tau(y), \text { with } \tau \in \mathcal{C}(V)
$$

and hence $\widetilde{G}_{y}<\frac{\sigma}{\sigma(y)} \widetilde{G}_{y}(y)$ on $V \backslash\{y\}$. In addition, the orthogonal Green kernel for $V$ is given by the expression

$$
G(x, y)=\frac{\sigma(y)}{\|\sigma\|_{2}^{4}}\left(\left\|\sigma u_{y}\right\| \sigma(x)-\|\sigma\|_{2}^{2} u_{y}(x)\right), \text { for all } x, y \in V
$$

and hence any symmetric Green kernel for $V$ is given by the expression $\widetilde{G}(x, y)=\frac{\left(b+\left\|\sigma u_{y}\right\|\right)}{\|\sigma\|_{2}^{4}} \sigma(x) \sigma(y)-\frac{1}{\|\sigma\|_{2}^{2}} u_{y}(x) \sigma(y), b \in \mathbb{R}$, for all $x, y \in V$.

Proof. By Proposition 5.8, if $\widetilde{G}$ is a Green kernel for $V$, then for all $y \in V$, $\widetilde{G}_{y}$ must satisfy that

$$
\mathcal{L}_{q_{\sigma}}\left(\widetilde{G}_{y}\right)=\varepsilon_{y}-\frac{\sigma(y)}{\|\sigma\|_{2}^{2}} \sigma, \quad \text { for all } y \in V
$$

Given $y \in V$ consider now $u_{y}$ the unique solution of the Dirichlet problem $\mathcal{L}_{q_{\sigma}}(u)=\sigma$ on $V \backslash\{y\}$ and $u(y)=0$. Then, $u_{y} \in \mathcal{C}^{*}(V \backslash\{y\})$ from Corollary 4.3 and applying the Second Green identity we obtain that

$$
0=\int_{V} u_{y} \mathcal{L}_{q_{\sigma}}(\sigma) d x=\int_{V} \sigma \mathcal{L}_{q_{\sigma}}\left(u_{y}\right) d x=\mathcal{L}_{q_{\sigma}}\left(u_{y}\right)(y) \sigma(y)+\|\sigma\|_{2}^{2}-\sigma(y)^{2}
$$

and hence $\mathcal{L}_{q_{\sigma}}\left(u_{y}\right)=\sigma-\frac{\|\sigma\|_{2}^{2}}{\sigma(y)} \varepsilon_{y}$. Therefore, the function

$$
\widehat{G}(x, y)=-\frac{\sigma(y)}{\|\sigma\|_{2}^{2}} u_{y}(x), \quad x, y \in V
$$

is a Green kernel for $V$. Moreover, $\widetilde{G}$ is another Green kernel for $V$ iff for all $y \in V, \widetilde{G}_{y}=\widehat{G}_{y}+\tau(y) \sigma$, where $\tau(y) \in \mathbb{R}$ and hence

$$
\frac{\sigma(x)}{\sigma(y)} \widetilde{G}(y, y)-\widetilde{G}(x, y)=\frac{\sigma(y)}{\|\sigma\|_{2}^{2}} u_{y}(x)>0 \quad \text { for } x \neq y
$$


Therefore, $\widetilde{G}_{y}<\frac{\sigma}{\sigma(y)} \widetilde{G}_{y}(y)$ on $V \backslash\{y\}$. In addition, the orthogonal Green kernel for $V$ is obtained by choosing $\tau(y)=\sigma(y)\|\sigma\|_{2}^{-4}\left\|\sigma u_{y}\right\|$ and the characterization of symmetric Green kernels follows from the last claim of Proposition 5.8.

Observe that the formula given in the above proposition for the Green kernels for $V$ associated with $\mathcal{L}_{q}$ does not depend on the choice of $\sigma \in \mathcal{C}^{*}(V)$ such that $q=q_{\sigma}$. In fact, if $\mu \in \mathcal{C}^{*}(V)$ is such that $q_{\mu}=q_{\sigma}$, then $\mu=a \sigma$, $a>0$ and hence for all $y \in V$ the unique solution of $\mathcal{L}_{q}(v)=\mu$ on $V \backslash\{y\}$, $v(y)=0$ is $v_{y}=a u_{y}$. Therefore,

$$
\frac{\sigma(y)}{\|\sigma\|_{2}^{4}}\left(\left\|\sigma u_{y}\right\| \sigma(x)-\|\sigma\|_{2}^{2} u_{y}(x)\right)=\frac{\mu(y)}{\|\mu\|_{2}^{4}}\left(\left\|\mu v_{y}\right\| \mu(x)-\|\mu\|_{2}^{2} v_{y}(x)\right)
$$

and both expressions determine the orthogonal Green kernel for $V$.

On the other hand, when $\sigma$ is a constant function, and hence $q=0$, then $u_{y}=\sigma \nu_{y}$ for all $y \in V$ and therefore the function $\widetilde{G}(x, y)=-\frac{1}{n} \nu_{y}(x)$, $x, y \in V$ determines a Green kernel for $V$, associated with the combinatorial Laplacian. Moreover any Green kernel for $V$ has the expression $\widetilde{G}(x, y)=$ $-\frac{1}{n} \nu_{y}(x)+\tau(y)$ for all $x, y \in V$ where $\tau \in \mathcal{C}(V)$ and the orthogonal Green kernel for $V$ associated with the combinatorial Laplacian is given by

$$
G(x, y)=\frac{1}{n^{2}}\left(\left\|\nu_{y}\right\|-n \nu_{y}(x)\right), \text { for all } x, y \in V .
$$

This formula was obtained in [4].

After the above proposition, to express the orthogonal Green kernel for $V$ in terms of equilibrium measures for the sets with cardinality $n-1$, it is enough to express each function $u_{y}$ by means of this type of measures. Since for all $y \in V$,

$$
u_{y}(x)=\int_{V} G^{V \backslash\{y\}}(x, z) \sigma(z) d z
$$

and from identity (6.1),

$$
G^{V \backslash\{y\}}(x, z)=\left(1-\mathcal{L}_{q_{\sigma}}\left(\nu^{V \backslash\{y, z\}}(z)\right)^{-1}\left(\nu_{y}(x)-\nu^{V \backslash\{y, z\}}(x)\right),\right.
$$

for all $x, y, z \in V$, it is enough to express $\nu^{V \backslash\{y, z\}}$ by means of equilibrium measures for the sets with cardinality $n-1$. For that we prove a more general result: if $q=q_{\sigma}$, the equilibrium measure for each proper subset $F$ can be expressed as a linear combination of $\sigma$ and the equilibrium measures for the sets $F \cup\{y\}$ with $y \in \delta(F)$. So, iterating the argument it follows that the equilibrium measure for $F$ can be obtained by means of a suitable linear combination of $\sigma$ and $\left\{\nu_{y}\right\}_{y \in \delta(F)}$. 
Proposition 6.11 Suppose that $q=q_{\sigma}$ and consider $F \subset V$ such that $1 \leq|F| \leq n-2$. Then,

$$
\nu^{F}=\left(\int_{\delta(F)} \frac{\sigma(y)}{\nu^{F \cup\{y\}}(y)} d y\right)^{-1}\left(\int_{\delta(F)} \frac{\sigma(y)}{\nu^{F \cup\{y\}}(y)} \nu^{F \cup\{y\}} d y-\sigma\right) 1_{\bar{F}} .
$$

In particular, for each $x, y \in V$ such that $x \neq y$, it is verified that

$$
\nu^{V \backslash\{x, y\}}=\left(\sigma(x) \nu_{x}(y)+\sigma(y) \nu_{y}(x)\right)^{-1}\left(\sigma(x) \nu_{x}(y) \nu_{y}+\sigma(y) \nu_{y}(x) \nu_{x}-\nu_{x}(y) \nu_{y}(x) \sigma\right) .
$$

Proof. As $\left|F^{c}\right| \geq 2$, then for each $y \in F^{c}, F \cup\{y\}$ is a proper subset and hence there exists the equilibrium measure for $F \cup\{y\}$. Consider now $\{A, B\}$ a partition of $F^{c}$ in such a way that $A$ and $B$ are non empty subsets. Also consider $u, v \in \mathcal{C}(V)$ the solutions of the following boundary value problems

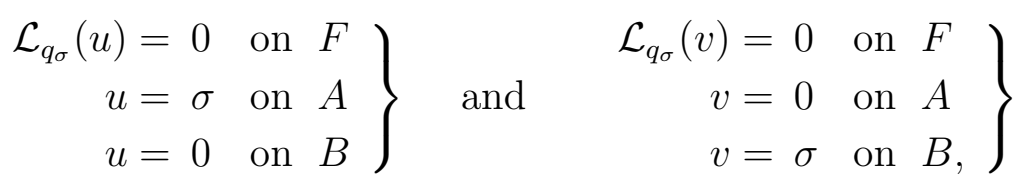

respectively. Then, from definition of the Poisson kernel for $F$, we have that

$$
\begin{aligned}
& u(x)=\int_{F^{c}} P^{F}(x, y) \sigma_{\left.\right|_{A}}(y) d y=\int_{A} P^{F}(x, y) \sigma(y) d y, \\
& v(x)=\int_{F^{c}} P^{F}(x, y) \sigma_{\left.\right|_{B}}(y) d y=\int_{B} P^{F}(x, y) \sigma(y) d y .
\end{aligned}
$$

Keeping in mind the expression for $P^{F}$ obtained in part (ii) of Proposition 6.7 we have that

$$
\begin{aligned}
& u=\int_{A} \frac{\sigma(y)}{\nu^{F \cup\{y\}}(y)} \nu^{F \cup\{y\}} d y-\left(\int_{A} \frac{\sigma(y)}{\nu^{F \cup\{y\}}(y)} d y\right) \nu^{F}, \\
& v=\int_{B} \frac{\sigma(y)}{\nu^{F \cup\{y\}}(y)} \nu^{F \cup\{y\}} d y-\left(\int_{B} \frac{\sigma(y)}{\nu^{F \cup\{y\}}(y)} d y\right) \nu^{F} .
\end{aligned}
$$

On the other hand, since $\mathcal{L}_{q_{\sigma}}(\sigma)=0$ it is clear that $u+v=\sigma$ and hence

$$
\sigma=\int_{F^{c}} \frac{\sigma(y)}{\nu^{F \cup\{y\}}(y)} \nu^{F \cup\{y\}} d y-\left(\int_{F^{c}} \frac{\sigma(y)}{\nu^{F \cup\{y\}}(y)} d y\right) \nu^{F} .
$$

Observe that when $y \notin \delta(F)$ then $\nu^{F \cup\{y\}}=\nu^{F}+\nu^{\{y\}}$, which implies that $\nu^{F \cup\{y\}}(y)=\nu^{\{y\}}(y)$ and

$$
\int_{\operatorname{Ext}(F)} \frac{\sigma(y)}{\nu^{F \cup\{y\}}(y)} \nu^{F \cup\{y\}} d y-\left(\int_{\operatorname{Ext}(F)} \frac{\sigma(y)}{\nu^{F \cup\{y\}}(y)} d y\right) \nu^{F}=\sigma 1_{\mathrm{Ext}(\mathrm{F})}
$$


and therefore,

$$
\nu^{F}=\left(\int_{\delta(F)} \frac{\sigma(y)}{\nu^{F \cup\{y\}}(y)} d y\right)^{-1}\left(\int_{\delta(F)} \frac{\sigma(y)}{\nu^{F \cup\{y\}}(y)} \nu^{F \cup\{y\}} d y-\sigma 1_{\bar{F}}\right) .
$$

In particular, when $F=V \backslash\{x, y\}$, then $F \cup\{y\}=V \backslash\{x\}, F \cup\{x\}=$ $V \backslash\{y\}$ and hence

$$
\begin{aligned}
\nu^{V \backslash\{x, y\}} & =\left(\frac{\sigma(y)}{\nu_{x}(y)}+\frac{\sigma(x)}{\nu_{y}(x)}\right)^{-1}\left(\frac{\sigma(y)}{\nu_{x}(y)} \nu_{x}+\frac{\sigma(x)}{\nu_{y}(x)} \nu_{y}-\sigma\right) \\
& =\frac{\nu_{x}(y) \nu_{y}(x)}{\sigma(x) \nu_{x}(y)+\sigma(y) \nu_{y}(x)}\left(\frac{\sigma(y)}{\nu_{x}(y)} \nu_{x}+\frac{\sigma(x)}{\nu_{y}(x)} \nu_{y}-\sigma\right) .
\end{aligned}
$$

Proposition 6.12 If $q=q_{\sigma}$, then for all $x, y, z \in V$ we have the following expressions

$$
\begin{aligned}
& G^{V \backslash\{y\}}(x, z)=\frac{1}{\|\sigma\|} \frac{\sigma(z)}{\sigma(y)}\left(\sigma(y) \nu_{y}(x)+\sigma(x) \nu_{z}(y)-\sigma(y) \nu_{z}(x)\right), \\
& P^{V \backslash\{y, z\}}(x, y)=\left(\sigma(z) \nu_{z}(y)+\sigma(y) \nu_{y}(z)\right)^{-1}\left(\sigma(z) \nu_{z}(x)+\sigma(x) \nu_{y}(z)-\sigma(z) \nu_{y}(x)\right), \\
& P^{V \backslash\{y, z\}}(x, z)=\left(\sigma(z) \nu_{z}(y)+\sigma(y) \nu_{y}(z)\right)^{-1}\left(\sigma(y) \nu_{y}(x)+\sigma(x) \nu_{z}(y)-\sigma(y) \nu_{z}(x)\right) .
\end{aligned}
$$

Proof. First, applying the above proposition, we have that for all $y, z \in V$ with $z \neq y$,

$$
\nu_{y}-\nu^{V \backslash\{y, z\}}=\left(\sigma(z) \nu_{z}(y)+\sigma(y) \nu_{y}(z)\right)^{-1} \nu_{y}(z)\left(\sigma(y)\left(\nu_{y}-\nu_{z}\right)+\nu_{z}(y) \sigma\right)
$$

and keeping in mind that $\mathcal{L}_{q_{\sigma}}\left(\nu_{z}\right)(z)=1-\frac{\|\sigma\|}{\sigma(z)}$ and $\mathcal{L}_{q_{\sigma}}\left(\nu_{y}\right)(z)=1$,

$$
1-\mathcal{L}_{q_{\sigma}}\left(\nu^{V \backslash\{y, z\}}\right)(z)=\frac{\sigma(y)}{\sigma(z)}\|\sigma\|\left(\sigma(z) \nu_{z}(y)+\sigma(y) \nu_{y}(z)\right)^{-1} \nu_{y}(z) .
$$

Finally, we obtain the formula for $G^{V \backslash\{y\}}$ by replacing the above identities in (6.1) and the formulae for the $P^{V \backslash\{y, z\}}$ by applying the relation between the Green and Poisson kernels given in Proposition 5.10.

The symmetry of the Green kernel for each set of the form $V \backslash\{y\}$ leads to the following result about the relation between the values $\nu_{y}(x)$ and $\nu_{x}(y)$.

Corollary 6.13 When $q=q_{\sigma}$, then

$$
\sigma(x) \nu_{x}(y)=\sigma(y) \nu_{y}(x)+\|\sigma\|^{-1} \sigma(x) \sigma(y)\left(\left\|\nu_{x}\right\|-\left\|\nu_{y}\right\|\right) \text { for all } x, y \in V .
$$

In particular, $\sigma(x) \nu_{x}(y)=\sigma(y) \nu_{y}(x)$ iff $\operatorname{cap}_{q_{\sigma}}(V \backslash\{x\})=\operatorname{cap}_{q_{\sigma}}(V \backslash\{y\})$. 
Proof. For fixed $y \in V$, by applying the formula for $G^{V \backslash\{y\}}$ given at the above proposition, for all $x \in V$ we get that

$\nu_{y}(x)=\int_{V} G^{V \backslash\{y\}}(x, z) d z=\nu_{y}(x)+\frac{1}{\|\sigma\|} \frac{1}{\sigma(y)} \int_{V} \sigma(z)\left(\sigma(x) \nu_{z}(y)-\sigma(y) \nu_{z}(x)\right) d z$

which implies that

$$
\int_{V} \sigma(z)\left(\sigma(x) \nu_{z}(y)-\sigma(y) \nu_{z}(x)\right) d z=0
$$

On the other hand, applying the symmetry of $G^{V \backslash\{y\}}$ we obtain that

$$
\begin{aligned}
\sigma(z)(\sigma(y) & \left.\nu_{y}(x)-\sigma(x) \nu_{x}(y)\right) \\
& =\sigma(x) \sigma(y)\left(\nu_{y}(z)-\nu_{x}(z)\right)+\sigma(z)\left(\sigma(y) \nu_{z}(x)-\sigma(x) \nu_{z}(y)\right)
\end{aligned}
$$

and hence the result follows by integrating with respect to $z$.

Observe that when $\sigma$ is a constant function, then the above formula becomes

$$
\nu_{x}(y)=\nu_{y}(x)+\frac{1}{n}\left(\left\|\nu_{x}\right\|-\left\|\nu_{y}\right\|\right) \quad \text { for all } x, y \in V .
$$

Of course, this identity can be directly obtained from the expression of the orthogonal Green kernel for $V$ associated with the combinatorial Laplacian.

Proposition 6.14 When $q=q_{\sigma}$, the orthogonal Green kernel for $V$ is given by

$$
\begin{aligned}
G(x, y) & =\alpha \sigma(x) \sigma(y) \int_{V} \sigma(z) \nu_{y}(z) d z-\|\sigma\|^{-1} \sigma(y) \nu_{y}(x) \\
& +\alpha \sigma(y) \int_{V} \sigma^{2}(z) \nu_{z}(x) d z-\beta \sigma(x) \sigma(y) \int_{V} \int_{V} \sigma^{2}(z) \sigma(w) \nu_{z}(w) d z d w
\end{aligned}
$$

where $\alpha=\|\sigma\|^{-1}\|\sigma\|_{2}^{-2}$ and $\beta=\|\sigma\|^{-1}\|\sigma\|_{2}^{-4}$.

Proof. From Proposition 6.10, we know that

$$
G(x, y)=\frac{\left\|\sigma u_{y}\right\|}{\|\sigma\|_{2}^{4}} \sigma(x) \sigma(y)-\frac{\sigma(y)}{\|\sigma\|_{2}^{2}} u_{y}(x),
$$

where $u_{y}(x)=\int_{V} G^{V \backslash\{y\}}(x, z) \sigma(z) d z$, for all $x, y \in V$. 
Applying the formula for $G^{V \backslash\{y\}}$ obtained in the above proposition we have that

$$
\begin{aligned}
u_{y}(x)= & \frac{\|\sigma\|_{2}^{2}}{\|\sigma\|} \nu_{y}(x)+\frac{1}{\|\sigma\|} \frac{\sigma(x)}{\sigma(y)} \int_{V} \nu_{z}(y) \sigma(z)^{2} d z-\frac{1}{\|\sigma\|} \int_{V} \nu_{z}(x) \sigma(z)^{2} d z \\
\left\|\sigma u_{y}\right\|= & \frac{\|\sigma\|_{2}^{2}}{\| \sigma{ }_{V}} \int_{V} \nu_{y}(z) \sigma(z) d z+\frac{\|\sigma\|_{2}^{2}}{\|\sigma\|} \frac{1}{\sigma(y)} \int_{V} \nu_{z}(y) \sigma(z)^{2} d z \\
& -\frac{1}{\|\sigma\|} \int_{V} \int_{V} \nu_{z}(w) \sigma(z)^{2} \sigma(w) d z d w
\end{aligned}
$$

and the expression for $G$ follows by replacing $u_{y}$ and $\left\|\sigma u_{y}\right\|$ in the formula given at the beginning of the proof.

The above formula allows us to obtain an expression of the effective resistance in terms of equilibrium measures. Recall that when talking about effective resistance we need to consider $\sigma$ as the unique normalized function such that $q=q_{\sigma}$. From now on, we consider that function $\sigma \in \mathcal{C}_{n}^{*}(V)$ and we suppose that $q=q_{\sigma}$.

Proposition 6.15 For all $x, y \in V$,

$$
R_{\sigma}(x, y)=\frac{1}{n}\left(\frac{\nu_{x}(y)}{\sigma(y)}+\frac{\nu_{y}(x)}{\sigma(x)}\right) .
$$

Proof. Given $x, y \in V$, if we consider $u=\sigma(x) P_{x}^{V \backslash\{x, y\}}$ then $\mathcal{L}_{q_{\sigma}}(u)=0$ on $V \backslash\{x, y\}, u(x)=\sigma(x), u(y)=0$ and hence $C_{\sigma}(x, y)=\sigma(x)^{2} \mathcal{L}_{q_{\sigma}}\left(P_{x}^{V \backslash\{x, y\}}\right)(x)$. Taking into account that

$$
P_{x}^{V \backslash\{x, y\}}=\left(\sigma(x) \nu_{x}(y)+\sigma(y) \nu_{y}(x)\right)^{-1}\left(\sigma(y) \nu_{y}+\sigma \nu_{x}(y)-\sigma(y) \nu_{x}\right),
$$

we obtain that

$$
\mathcal{L}_{q_{\sigma}}\left(P_{x}^{V \backslash\{x, y\}}\right)=n\left(\sigma(x) \nu_{x}(y)+\sigma(y) \nu_{y}(x)\right)^{-1} \sigma(y) \frac{1}{\sigma}\left(\varepsilon_{x}-\varepsilon_{y}\right),
$$

which implies that $C_{\sigma}(x, y)=n\left(\sigma(x) \nu_{x}(y)+\sigma(y) \nu_{y}(x)\right)^{-1} \sigma(y) \sigma(x)$.

The above formula for $R_{\sigma}$ generalizes the one obtained in [5], when $q=0$. On the other hand, we next obtain a generalization of the so-called Foster's Theorem, whose proof is obtained directly from the expression of the effective resistance. For other type of proofs, see for instance $[17,18]$. 
Corollary 6.16 (Foster's Theorem) If $q=q_{\sigma}$, then it is verified that

$$
\int_{V} \int_{V} R_{\sigma}(x, y) c(x, y) \sigma(x) \sigma(y) d x d y=2(n-1) .
$$

Proof. First, observe that

$$
\int_{V} \int_{V} \sigma(x) \nu_{x}(y) c(x, y) d x d y=\int_{V} \int_{V} \sigma(y) \nu_{y}(x) c(x, y) d x d y,
$$

since $c$ is a symmetric function. Taking into account that

$$
R_{\sigma}(x, y) \sigma(x) \sigma(y)=n^{-1}\left(\sigma(x) \nu_{x}(y)+\sigma(y) \nu_{y}(x)\right)
$$

we obtain

$$
\int_{V} \int_{V} R_{\sigma}(x, y) c(x, y) \sigma(x) \sigma(y) d x d y=\frac{2}{n} \int_{V} \sigma(x)\left(\int_{V} \nu_{x}(y) c(x, y) d y\right) d x .
$$

On the other hand, since

$$
\int_{V} \nu_{x}(y) c(x, y) d x=-\mathcal{L}_{q_{\sigma}}\left(\nu_{x}\right)(x)=\frac{n}{\sigma(x)}-1,
$$

the right hand side of the above identity equals $\frac{2}{n} \int_{V}(n-\sigma) d x=2(n-1)$.

Let $x, y, z \in V$, then we say that $z$ separates $x$ and $y$ iff the set $V \backslash\{z\}$ is not connected and $x$ and $y$ belong to different connected components of it. The following formula enables us to express the Green kernel for subsets with cardinality $n-1$ by means of effective resistances. In its standard version, it can be found in $[16,17,18]$.

Corollary 6.17 If $q=q_{\sigma}$, then for all $x, y, z \in V$ it is verified that

$$
G^{V \backslash\{z\}}(x, y)=\frac{1}{2} \sigma(x) \sigma(y)\left(R_{\sigma}(x, z)+R_{\sigma}(x, y)-R_{\sigma}(x, y)\right) .
$$

In particular, $R_{\sigma}$ defines a distance on $V$. Moreover $R_{\sigma}(x, y)=R_{\sigma}(x, z)+$ $R_{\sigma}(z, y)$ iff $z$ separates $x$ and $y$.

Proof. From Proposition 6.12 we know that

$$
\begin{aligned}
& G^{V \backslash\{z\}}(x, y)=\frac{1}{n} \sigma(x) \sigma(y)\left(\frac{\nu_{z}(x)}{\sigma(x)}+\frac{\nu_{y}(z)}{\sigma(z)}-\frac{\nu_{y}(x)}{\sigma(x)}\right), \\
& G^{V \backslash\{z\}}(y, x)=\frac{1}{n} \sigma(x) \sigma(y)\left(\frac{\nu_{z}(y)}{\sigma(y)}+\frac{\nu_{x}(z)}{\sigma(z)}-\frac{\nu_{x}(y)}{\sigma(y)}\right)
\end{aligned}
$$

and the first claim follows adding both sides of the above equalities and taking into account that $G^{V \backslash\{z\}}$ is symmetric. 
On the other hand, the symmetry of $R_{\sigma}$ and the nonnegativity of the Green kernels imply that the effective resistance is a distance on the vertex set. Moreover $R_{\sigma}(x, y)=R_{\sigma}(x, z)+R_{\sigma}(z, y)$, that is the triangle inequality is an equality, iff $G_{y}^{V \backslash\{z\}}(x)=0$. From Proposition 5.10, this condition is equivalent to the fact that $x$ and $y$ are in different connected components of $V \backslash\{z\}$.

\section{References}

[1] Ancona, A.: Continuité des contractions dans les espaces de Dirichlet. In Séminaire de Théorie du Potentiel de Paris, No. 2 (Univ. Paris, Paris, 1975-1976), 1-26. Lecture Notes in Math. 563. Springer, Berlin, 1976.

[2] Barlow, M. T. Diffusions on fractals. In Lectures on probability theory and statistics (Saint-Flour, 1995), 1-121. Lecture Notes in Math. 1690. Springer, Berlin, 1998.

[3] Bendito, E., Carmona, A. And Encinas, A. M.: Shortest paths in distance-regular graphs. European J. Combin. 21 (2000), 153-166.

[4] Bendito, E., Carmona, A. And Encinas, A. M.: Solving boundary value problems on networks using equilibrium measures. J. Funct. Anal. 171 (2000), 155-176.

[5] Bendito, E., Carmona, A. And Encinas, A. M.: Equilibrium measure, Poisson kernel and effective resistance on networks. In Random walks and geometry, 363-376. Walter de Gruyter, Berlin, 2004.

[6] Berman, A. And Plemons, R. J.: Nonnegative matrices in the mathematical sciences. Classics in Applied Mathematics 9. Society for Industrial and Applied Mathematics (SIAM), Philadelphia, PA, 1994.

[7] Biggs, N.: Algebraic potential theory on graphs. Bull. London Math. Soc. 29 (1997), 641-682.

[8] Beurling, A. And Deny, J.: Espaces de Dirichlet. I. Le cas élémentaire. Acta Math. 99 (1958), 203-224.

[9] Chung, F. R. K.: Spectral graph theory. CBMS Regional Conference Series in Mathematics 92. Amer. Math. Soc., Providence, RI, 1997.

[10] Chung, F. R. K. and Yau, S. T.: Discrete Green's functions. J. Combin. Theory Ser. A 91 (2000), 191-214.

[11] Colin de Verdière, Y.: Spectre d'opérateurs différentiels sur les graphes. In Random walks and discrete potential theory (Cortona, 1997), 139-164, Sympos. Math. 39. Cambridge Univ. Press, Cambridge, 1999.

[12] Dodziuk, J. And Karp, L.: Spectral and function theory for combinatorial Laplacians. In Geometry of random motion (Ithaca, N.Y., 1987), 25-40. Contemp. Math. 73, Amer. Math. Soc., Providence, RI, 1988. 
[13] Gilbarg, D. And Trudinger, N.S.: Elliptic partial differential equations of second order. Classics in Mathematics. Springer-Verlag, Berlin, 2001.

[14] Kigami, J.: Analysis on fractals. Cambridge Tracts in Math. 143. Cambridge University Press, Cambridge, 2001.

[15] Kayano, T. and Yamasaki, M.: Discrete Dirichlet integral formula. Discrete Appl. Math. 22 (1988/89), 53-68.

[16] Metz, V.: Shorted operators: an application in potential theory. Linear Algebra Appl. 264 (1997), 439-455.

[17] Ponzio, S.: The combinatorics of effective resistances and resistive inverses. Inform. and Comput. 147 (1998), 209-223.

[18] Tetali, P.: An extension of Foster's network theorem. Combin. Probab. Comput. 3 (1994), 421-427.

[19] Urakawa, H.: A discrete analogue of the harmonic morphism and Green kernel comparison theorems. Glasg. Math. J. 42 (2000), 319-334.

[20] Yamasaki, M.: The equation $\Delta u=q u$ on an infinite network. Mem. Fac. Sci. Shimane Univ. 21 (1987), 31-46.

Recibido: 4 de marzo de 2003

Revisado: 5 de marzo de 2004

Enrique Bendito

Departament de Matemàtica Aplicada III

Universitat Politècnica de Catalunya

08034 Barcelona

enrique.bendito@upc.edu

Ángeles Carmona

Departament de Matemàtica Aplicada III

Universitat Politècnica de Catalunya

08034 Barcelona

angeles.carmona@upc.edu

Andrés M. Encinas

Departament de Matemàtica Aplicada III

Universitat Politècnica de Catalunya

08034 Barcelona

andres.marcos.encinas@upc.edu

This work has been partly supported by the Spanish Research Council (Comisión Interministerial de Ciencia y Tecnología) under projects BFM2000-1063 and BFM2003-06014. 